\title{
Hydrogen Bond Catalyzed Enantioselective Vinylogous Mukaiyama Aldol Reaction
}

\author{
Vijaya Bhasker Gondi, Michel Gravel, and Viresh H. Rawal* \\ Department of Chemistry, The University of Chicago \\ 5735 South Ellis Avenue, Chicago, IL 60637
}

\section{Organic Letters}

\section{Supporting Information}

General Information................................................................................................ 2

General Procedure for the VMA Reaction.................................................................... 3

Characterization Data for VMA Products 3a-1 .......................................................... 4

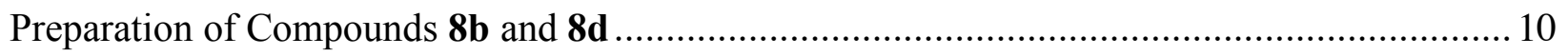

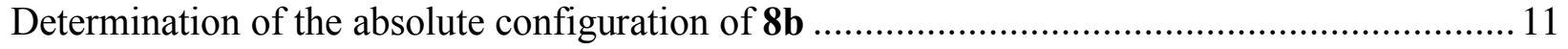

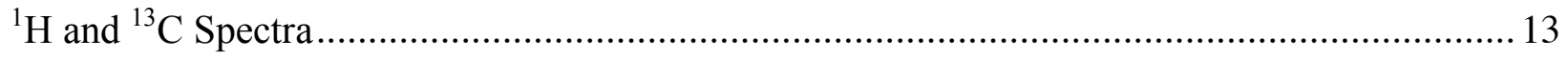

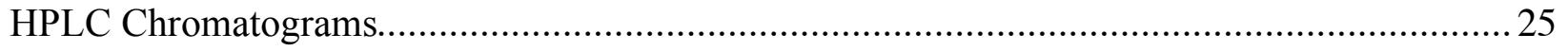

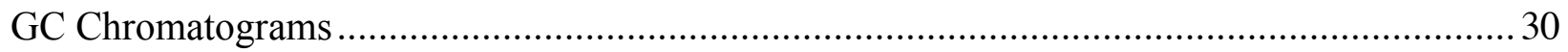




\section{General Information}

Unless otherwise noted, all solvents were degassed by purging with argon and dried over activated alumina. All reactions were performed under argon with either flame or oven dried glassware. $\mathrm{N}, \mathrm{N}$-disopropylethylamine was distilled over $\mathrm{CaH}_{2}$. Ethyl glyoxalate was purchased as a solution in toluene (Fluka), and was purified by bulb-to-bulb distillation, giving a 39\% wt. solution in toluene. Benzyloxyacetaldehyde was purchased from Aldrich and purified by bulbto-bulb distillation prior to use. The silyldienol ethers were prepared according to a known procedure $^{1}$ and were bulb-to-bulb distilled prior to use. Catalyst $\mathbf{5}$ was obtained from Dr. Aditya K. Unni. ${ }^{2}$ Melting points were measured on a Thomas Hoover melting point apparatus and are uncorrected. ${ }^{1} \mathrm{H}$ and ${ }^{13} \mathrm{C}$ MNR were recorded on Bruker DRX-400 or $500 \mathrm{MHz}$ spectrometers. Proton chemical shifts are internally referenced to the residual solvent proton resonance $\left(\mathrm{CHCl}_{3}\right.$ $\delta$ 7.26). Carbon chemical shifts are internally referenced to the deuterated solvent signal $\left(\mathrm{CDCl}_{3}\right.$ $\delta$ 77.36). Infrared spectra were obtained on a Nicolet 20 SXB FT-IR spectrometer. Electrospray ionization high resolution mass spectra were recorded at the Ohio State University. Chemical ionization mass spectra were recorded on an Agilent 1100 mass spectrometer. Optical rotations were recorded using a Jasco DIP-1000 instrument. Analytical thin layer chromatography was run on Whatman $0.25 \mathrm{~mm} \mathrm{K6F}$ silica gel $60 \AA$ plates. Flash chromatography was run using silica gel (60 ̊, 230-400 mesh) obtained from Silicycle and used as received. High pressure liquid chromatography was performed on an Agilent 1100 system using a Chiralcel OD-H column and a $254 \mathrm{~nm}$ UV detector. Gas chromatography was performed on an Agilent 6850 system using a Cyclosilb chiral column.

\footnotetext{
${ }^{1}$ Denmark, S.E.; Beutner, G.L.; Wynn, T.; Eastgate, M.D. J. Am. Chem. Soc. 2005, 127, 3774.

${ }^{2}$ Unni, A. K. Ph.D. Thesis, The University of Chicago, June 2005.
} 


\section{General Procedure for the VMA Reaction}

Products 3b and 3c: To a solution of (4S-trans)-2,2-dimethyl- $\alpha, \alpha, \alpha^{\prime}, \alpha^{\prime}$-tetra(1-naphthyl)-1,3dioxolane-4,5-dimethanol (1) $(40 \mathrm{mg}, 0.06 \mathrm{mmol})$ in $1.5 \mathrm{~mL}$ of toluene at $-78{ }^{\circ} \mathrm{C}$ were added sequentially $N, N$-disopropylethylamine $(5 \mu \mathrm{L}, 0.03 \mathrm{mmol})$, the aldehyde $(0.6 \mathrm{mmol})$, and the silyl dienol ether $(0.3 \mathrm{mmol})$ dropwise. The reaction mixture was then stirred at the indicated temperature for the time indicated in Table 3 . The reaction was quenched by adding $1 \mathrm{~N} \mathrm{HCl}(2$ $\mathrm{mL}$ ) and stirring at room temperature for 15 minutes, then neutralized with a saturated aqueous solution of $\mathrm{NaHCO}_{3}$. The aqueous layer was extracted with $\mathrm{Et}_{2} \mathrm{O}(2 \times 3 \mathrm{~mL})$. The organic layers were combined, dried over $\mathrm{MgSO}_{4}$, and concentrated in vacuo. The crude residue was purified by flash column chromatography to afford the products $\mathbf{3 b}$ and $\mathbf{3 c}$.

Products 3a,d-l: To a solution of (4S-trans)-2,2-dimethyl- $\alpha, \alpha, \alpha^{\prime}, \alpha^{\prime}$-tetra(1-naphthyl)-1,3dioxolane-4,5-dimethanol (7) $(40 \mathrm{mg}, 0.06 \mathrm{mmol})$ in $1.5 \mathrm{~mL}$ of toluene at $-78{ }^{\circ} \mathrm{C}$ were added sequentially the aldehyde $(0.3 \mathrm{mmol})$, and the silyl dienol ether $(0.6 \mathrm{mmol})$ dropwise. The reaction mixture was then stirred at the indicated temperature for the required time according to Table 2 . The reaction was quenched by adding $1 \mathrm{~N} \mathrm{HCl}(2 \mathrm{~mL})$ and stirring at room temperature for 15 minutes, then neutralized with a saturated aqueous solution of $\mathrm{NaHCO}_{3}$. The aqueous layer was extracted with $\mathrm{Et}_{2} \mathrm{O}(2 \times 3 \mathrm{~mL})$. The organic layers were combined, dried over $\mathrm{MgSO}_{4}$,

and concentrated in vacuo. The crude residue was purified by flash column chromatography to afford the products $\mathbf{3 a}, \mathbf{d}-\mathbf{l}$. 


\section{Characterization Data for VMA Products 3a-I}

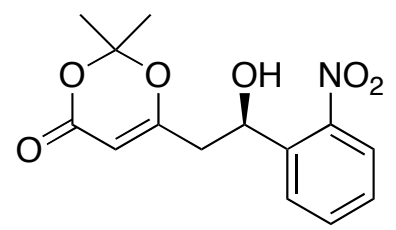

\section{(R)-6-(2-hydroxy-2-(2-nitrophenyl)ethyl)-2,2-dimethyl-4H-1,3-dioxin-4-one (3a)}

White solid. $\operatorname{mp~} 83-85^{\circ} \mathrm{C} ; \mathrm{Rf}=0.30(50 \%$ EtOAc/Hex $) ; \quad[\alpha]_{\mathrm{D}}^{25}+25.0^{\circ}\left(c=1.30, \mathrm{CHCl}_{3}\right) ; \quad \mathrm{IR}$ (neat, $\left.\mathrm{cm}^{-1}\right) 3433,3001,2944,1707,1634,1277 ;{ }^{1} \mathrm{H} \mathrm{NMR}\left(500 \mathrm{MHz}, \mathrm{CDCl}_{3}\right) \delta 7.98(\mathrm{~d}, J=7.8$ $\mathrm{Hz}, 1 \mathrm{H}), 7.88(\mathrm{~d}, J=7.8 \mathrm{~Hz}, 1 \mathrm{H}), 7.70,(\mathrm{t}, J=7.8 \mathrm{~Hz}, 1 \mathrm{H}), 7.48(\mathrm{t}, J=7.8 \mathrm{~Hz}, 1 \mathrm{H}), 5.57(\mathrm{dd}, J=$ 9.2, $3.0 \mathrm{~Hz}, 1 \mathrm{H}), 5.41(\mathrm{~s}, 1 \mathrm{H}), 2.85(\mathrm{dd}, J=14.8,3.1 \mathrm{~Hz}, 1 \mathrm{H}), 2.79$ (br s, 1H), $2.62(\mathrm{dd}, J=14.8$, $9.3 \mathrm{~Hz}, 1 \mathrm{H}), 1.71(\mathrm{~s}, 3 \mathrm{H}), 1.68(\mathrm{~s}, 3 \mathrm{H}) ;{ }^{13} \mathrm{C} \mathrm{NMR}\left(125 \mathrm{MHz}, \mathrm{CDCl}_{3}\right) \delta 168.6,161.5,147.5$, 138.8, 134.1, 128.9, 128.2, 124.7, 107.0, 95.4, 66.7, 42.4, 25.4, 24.7; HRMS (ESI, $m / z)$ calcd for $\mathrm{C}_{14} \mathrm{H}_{15} \mathrm{NO}_{6} \mathrm{Na}^{+}$316.079157, found 316.07938; HPLC: $20 \% i$ PrOH/Hexanes, $0.8 \mathrm{~mL} / \mathrm{min} ., 8.8$ min. (major), 11.7 min. (minor).<smiles>CCOC(=O)[C@H](O)CC1=CC(=O)OC(C)(C)O1</smiles>

\section{(R)-Ethyl 3-(2,2-dimethyl-4-oxo-4H-1,3-dioxin-6-yl)-2-hydroxypropanoate (3b)}

Colorless oil.; $\mathrm{Rf}=0.40(50 \%$ EtOAc/Hex $) ;[\alpha]_{\mathrm{D}}^{25}+34.0^{\circ}\left(c=1.55, \mathrm{CHCl}_{3}\right) ;$ IR (neat, $\left.\mathrm{cm}^{-1}\right)$ 3446, 2996, 1733, 1638, 1275; ${ }^{1} \mathrm{H}$ NMR (400 MHz, $\left.\mathrm{CDCl}_{3}\right) \delta 5.33(\mathrm{~s}, 1 \mathrm{H}), 4.38(\mathrm{t}, J=5.5 \mathrm{~Hz}$, 1H), $4.26(\mathrm{q}, J=7.0 \mathrm{~Hz}, 2 \mathrm{H}), 3.10($ br s, $1 \mathrm{H}), 2.75(\mathrm{dd}, J=15.0,5.0 \mathrm{~Hz}, 1 \mathrm{H}), 2.63(\mathrm{dd}, J=15.0$, $6.5 \mathrm{~Hz}, 1 \mathrm{H}), 1.67(\mathrm{~s}, 3 \mathrm{H}), 1.66(\mathrm{~s}, 3 \mathrm{H}), 1.31(\mathrm{t}, J=7.0 \mathrm{~Hz}, 3 \mathrm{H}) ;{ }^{13} \mathrm{C} \mathrm{NMR}\left(125 \mathrm{MHz}, \mathrm{CDCl}_{3}\right) \delta$ 
173.6, 167.1, 161.0, 107.0, 96.2, 67.3, 62.4, 38.2, 25.2, 24.9, 14.3; HRMS (ESI, $m / z$ ) calcd for $\mathrm{C}_{11} \mathrm{H}_{16} \mathrm{O}_{6} \mathrm{Na}^{+} 267.083908$, found 267.08341; HPLC: $20 \% i \mathrm{PrOH} / \mathrm{Hexanes}, 0.8 \mathrm{~mL} / \mathrm{min}$., $7.7 \mathrm{~min}$. (major), 9.2 min. (minor).

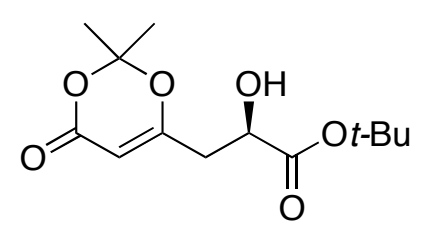

\section{(R)-tert-Butyl 3-(2,2-dimethyl-4-0xo-4H-1,3-dioxin-6-yl)-2-hydroxypropanoate (3c)}

Colorless oil.; $\mathrm{Rf}=0.22(30 \% \mathrm{EtOAc} / \mathrm{Hex}) ;[\alpha]_{\mathrm{D}}^{25}+34.1^{\circ}\left(c=0.22, \mathrm{CHCl}_{3}\right) ; \mathrm{IR}$ (neat, $\left.\mathrm{cm}^{-1}\right)$ 3454, 2981, 1733, 1638, 1274; ${ }^{1} \mathrm{H}$ NMR (400 MHz, $\left.\mathrm{CDCl}_{3}\right) \delta 5.34$ (s, 1H), 4.26 (q, $J=5.0 \mathrm{~Hz}$, $1 \mathrm{H}), 3.01(\mathrm{~d}, J=4.7 \mathrm{~Hz}, 1 \mathrm{H}), 2.73(\mathrm{ddd}, J=14.9,5.1,0.6 \mathrm{~Hz}, 1 \mathrm{H}), 2.60(\mathrm{ddd}, J=14.9,6.2,0.6$ $\mathrm{Hz}, 1 \mathrm{H}), 1.69$ (s, 3H), 1.68 (s, 3H), $1.50(\mathrm{~s}, 9 \mathrm{H}) ;{ }^{13} \mathrm{C} \mathrm{NMR}\left(100 \mathrm{MHz}, \mathrm{CDCl}_{3}\right) \delta$ 174.1, 168.7, 162.2, 107.9, 97.0, 84.4, 68.1, 38.7, 28.5, 25.7, 25.3; HRMS (ESI, $m / z$ ) calcd for $\mathrm{C}_{13} \mathrm{H}_{20} \mathrm{O}_{6} \mathrm{Na}^{+}$ 295.115208, found 295.11506; HPLC: 20\% $i \mathrm{PrOH} / \mathrm{Hexanes,} 0.8 \mathrm{~mL} / \mathrm{min}$., $6.1 \mathrm{~min}$. (major), 7.4 $\min$. (minor).

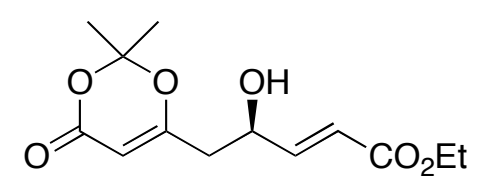

\section{(R,E)-ethyl 5-(2,2-dimethyl-4-oxo-4H-1,3-dioxin-6-yl)-4-hydroxypent-2-enoate (3d)}

White solid. $\mathrm{mp} 67-68^{\circ} \mathrm{C} ; \mathrm{Rf}=0.33(50 \%$ EtOAc/Hex $) ;[\alpha]^{25}{ }_{\mathrm{D}}+23.2^{\circ}\left(c=1.25, \mathrm{CHCl}_{3}\right) ; \quad \mathrm{IR}$ (neat, $\left.\mathrm{cm}^{-1}\right) 3432,2996,1717,1635,1276 ;{ }^{1} \mathrm{H}$ NMR $\left(500 \mathrm{MHz}, \mathrm{CDCl}_{3}\right) \delta 6.92(\mathrm{dd}, J=15.8,4.8$ $\mathrm{Hz}, 1 \mathrm{H}), 6.10(\mathrm{dd}, J=15.8,1.8 \mathrm{~Hz}, 1 \mathrm{H}), 5.35(\mathrm{~s}, 1 \mathrm{H}), 4.65-4.57(\mathrm{~m}, 1 \mathrm{H}), 4.20$ (q, $J=7.0 \mathrm{~Hz}$, 
2H), $2.58(\mathrm{~d}, J=5.0 \mathrm{~Hz}, 1 \mathrm{H}), 2.54(\mathrm{dd}, J=14.5,4.5 \mathrm{~Hz}, 1 \mathrm{H}), 2.45(\mathrm{dd}, J=14.5,8.5 \mathrm{~Hz}, 1 \mathrm{H})$, $1.69(\mathrm{~s}, 6 \mathrm{H}), 1.29(\mathrm{t}, J=7.0 \mathrm{~Hz}, 3 \mathrm{H}) ;{ }^{13} \mathrm{C} \mathrm{NMR}\left(125 \mathrm{MHz}, \mathrm{CDCl}_{3}\right) \delta 167.7,166.2,161.2,147.8$, 121.6, 107.1, 95.8, 68.0, 60.9, 40.8, 25.4, 25.0, 14.3; HRMS (ESI, $m / z$ ) calcd for $\mathrm{C}_{13} \mathrm{H}_{18} \mathrm{O}_{6} \mathrm{Na}^{+}$ 293.09987, found 293.09987; HPLC: 20\% iPrOH/Hexanes, $0.8 \mathrm{~mL} / \mathrm{min} ., 9.2 \mathrm{~min}$. (major), 11.2 $\min$. (minor).

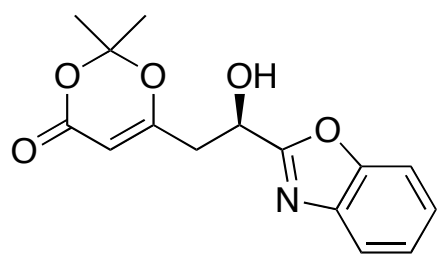

(R)-6-(2-(benzo[d] oxazol-2-yl)-2-hydroxyethyl)-2,2-dimethyl-4H-1,3-dioxin-4-one (3e)

Yellow solid. mp 138-140 ${ }^{\circ} \mathrm{C} ; \mathrm{Rf}=0.30(50 \% \mathrm{EtOAc} / \mathrm{Hex}) ;[\alpha]_{\mathrm{D}}^{25}+136.2^{\circ}\left(c=1.64, \mathrm{CHCl}_{3}\right)$; IR $\left(\right.$ neat, $\left.\mathrm{cm}^{-1}\right) 3363,3061,2990,1686,1634,1203 ;{ }^{1} \mathrm{H}$ NMR $\left(500 \mathrm{MHz}, \mathrm{CDCl}_{3}\right) \delta$ 7.72-7.68 (m, 1H), 7.55-7.52 (m, 1H), 7.40-7.32 (m, 1H), $5.41(\mathrm{~s}, 1 \mathrm{H}), 5.28-5.24(\mathrm{~m}, 1 \mathrm{H}), 3.88(\mathrm{~d}, J=5.0$ Hz, 1H), 3.02 (dd, $J=15.0,5.0 \mathrm{~Hz}, 1 \mathrm{H}), 2.93$ (dd, $J=15.0,8.0 \mathrm{~Hz}, 1 \mathrm{H}), 1.61$ (s, 3H), 1.59 (s, $3 \mathrm{H}) ;{ }^{13} \mathrm{C} \mathrm{NMR}\left(125 \mathrm{MHz}, \mathrm{CDCl}_{3}\right) \delta 166.7,165.9,161.0,151.0,140.3,125.9,125.1,120.2$, 111.0, 107.1, 96.3, 65.0, 39.6, 25.2, 25.0; HRMS (ESI, m/z) calcd for $\mathrm{C}_{15} \mathrm{H}_{15} \mathrm{NO}_{5} \mathrm{Na}^{+}$ 312.084242, found 312.08432; HPLC: $20 \% \mathrm{iPrOH} / \mathrm{Hexanes,} 0.8 \mathrm{~mL} / \mathrm{min}$., $10.4 \mathrm{~min}$. (major), 17.5 min. (minor).

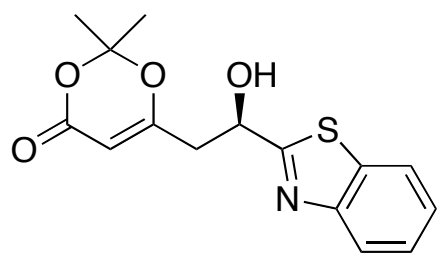




\section{(R)-6-(2-(benzo[d] thiazol-2-yl)-2-hydroxyethyl)-2,2-dimethyl-4H-1,3-dioxin-4-one (3f)}

Yellow oil. $\mathrm{Rf}=0.38(50 \%$ EtOAc/Hex $) ;[\alpha]_{\mathrm{D}}^{25}+59.2^{\circ}\left(c=0.99, \mathrm{CHCl}_{3}\right) ; \quad \mathrm{IR}\left(\right.$ neat, $\left.\mathrm{cm}^{-1}\right) 3393$, 3019, 1716, 1636, 1215; ${ }^{1} \mathrm{H}$ NMR $\left(500 \mathrm{MHz}, \mathrm{CDCl}_{3}\right) \delta 7.95(\mathrm{~d}, J=8.0 \mathrm{~Hz}, 1 \mathrm{H}), 7.89(\mathrm{~d}, J=8.0$ Hz, 1H), 7.48 (t, $J=8.0 \mathrm{~Hz}, 1 \mathrm{H}), 7.39$ (t, $8.0 \mathrm{~Hz}, 1 \mathrm{H}), 5.39(\mathrm{~s}, 1 \mathrm{H}), 5.39-5.37(\mathrm{~m}, 1 \mathrm{H}), 4.25-4.23$ (m, 1H), $3.05(\mathrm{dd}, J=14.8,3.8 \mathrm{~Hz}, 1 \mathrm{H}), 2.81(\mathrm{dd}, J=14.8,8.8 \mathrm{~Hz}, 1 \mathrm{H}), 1.66(\mathrm{~s}, 3 \mathrm{H}), 1.63(\mathrm{~s}$, $3 \mathrm{H}) ;{ }^{13} \mathrm{C}$ NMR $\left(125 \mathrm{MHz}, \mathrm{CDCl}_{3}\right) \delta 174.4,167.7,161.4,152.9,135.0,126.5,125.5,123.1$, 122.1, 107.1, 96.0, 69.2, 41.9, 25.3, 24.9; HRMS (ESI, $m / z$ ) calcd for $\mathrm{C}_{15} \mathrm{H}_{15} \mathrm{NO}_{4} \mathrm{SNa}^{+}$ 328.061397, found 328.06120; HPLC: 20\% iPrOH/Hexanes, $0.8 \mathrm{~mL} / \mathrm{min} ., 11.6 \mathrm{~min}$. (major), 26.5 min. (minor).

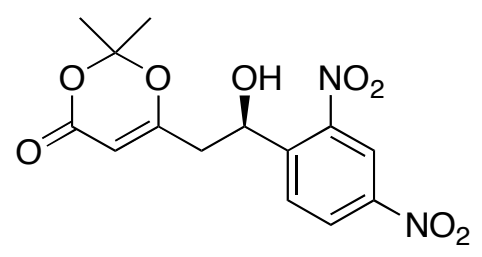

\section{(R)-6-(2-(2,4-dinitrophenyl)-2-hydroxyethyl)-2,2-dimethyl-4H-1,3-dioxin-4-one (3g)}

Yellow oil. $\mathrm{Rf}=0.40\left(50 \%\right.$ EtOAc/Hex); $[\alpha]_{\mathrm{D}}^{25}-13.4^{\circ}\left(c=0.95, \mathrm{CHCl}_{3}\right) ; \quad \mathrm{IR}$ (neat, $\left.\mathrm{cm}^{-1}\right) 3404$, 3112, 3003, 1708, 1635, 1204; ${ }^{1} \mathrm{H}$ NMR $\left(500 \mathrm{MHz}, \mathrm{CDCl}_{3}\right) \delta 8.83(\mathrm{~d}, J=2.0 \mathrm{~Hz}, 1 \mathrm{H}), 8.51(\mathrm{dd}$, $J=8.5,2.0 \mathrm{~Hz}, 1 \mathrm{H}), 8.21(\mathrm{~d}, J=8.5 \mathrm{~Hz}, 1 \mathrm{H}), 5.69(\mathrm{~d}, J=8.0 \mathrm{~Hz}, 1 \mathrm{H}), 5.42(\mathrm{~s}, 1 \mathrm{H}), 3.65$ (br s, 1H), $2.83(\mathrm{dd}, J=14.5,3.4 \mathrm{~Hz}, 1 \mathrm{H}), 2.55(\mathrm{dd}, J=14.5,9.3 \mathrm{~Hz}, 1 \mathrm{H}), 1.72(\mathrm{~s}, 3 \mathrm{H}), 1.71(\mathrm{~s}, 3 \mathrm{H})$; ${ }^{13} \mathrm{C}$ NMR $\left(125 \mathrm{MHz}, \mathrm{CDCl}_{3}\right) \delta 168.1,161.7,147.4,147.2,145.6,130.3,128.0,120.3,107.3$, 95.6, 66.6, 42.5, 25.4, 24.9; HRMS (ESI, $m / z$ ) calcd for $\mathrm{C}_{14} \mathrm{H}_{14} \mathrm{~N}_{2} \mathrm{O}_{8} \mathrm{Na}^{+}$361.064236, found 361.06450; HPLC: 20\% iPrOH/Hexanes, $0.8 \mathrm{~mL} / \mathrm{min} ., 17.9$ min. (major), $25.7 \mathrm{~min}$. (minor). 


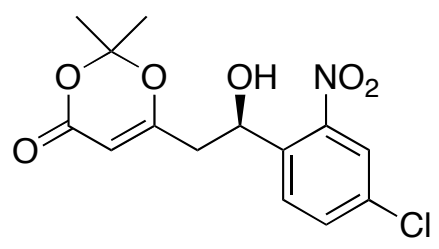

(R)-6-(2-(4-chloro-2-nitrophenyl)-2-hydroxyethyl)-2,2-dimethyl-4H-1,3-dioxin-4-one (3h)

Yellow oil. $\mathrm{Rf}=0.40(50 \% \mathrm{EtOAc} / \mathrm{Hex}) ;[\alpha]_{\mathrm{D}}^{25}-18.6^{\circ}\left(c=0.97, \mathrm{CHCl}_{3}\right) ; \mathrm{IR}\left(\right.$ neat, $\left.\mathrm{cm}^{-1}\right) 3415$, 3001, 2925, 1710, 1635, 1277; ${ }^{1} \mathrm{H}$ NMR (500 MHz, $\left.\mathrm{CDCl}_{3}\right) \delta 7.98(\mathrm{~d}, J=2.0 \mathrm{~Hz}, 1 \mathrm{H}), 7.86(\mathrm{~d}$, $J=8.5 \mathrm{~Hz}, 1 \mathrm{H}), 7.66(\mathrm{dd}, J=8.5,2.0 \mathrm{~Hz}, 1 \mathrm{H}), 5.57-5.53(\mathrm{~m}, 1 \mathrm{H}), 5.40(\mathrm{~s}, 1 \mathrm{H}), 3.04(\mathrm{~d}, J=4.0$ Hz, 1H), 2.81 (dd, $J=14.8,2.8 \mathrm{~Hz}, 1 \mathrm{H}), 2.56(\mathrm{dd}, J=14.8,9.3 \mathrm{~Hz}, 1 \mathrm{H}), 1.71(\mathrm{~s}, 3 \mathrm{H}), 1.69$ (s, $3 \mathrm{H}) ;{ }^{13} \mathrm{C}$ NMR $\left(125 \mathrm{MHz}, \mathrm{CDCl}_{3}\right) \delta 168.2,161.4,147.7,137.4,134.7,134.2,129.7,124.8$, 107.1, 95.6, 66.5, 42.5, 25.4, 24.9; HRMS (ESI, $m / z$ ) calcd for $\mathrm{C}_{14} \mathrm{H}_{14} \mathrm{ClNO}_{6} \mathrm{Na}^{+} 350.040184$, found 350.03986; HPLC: 20\% $i \mathrm{PrOH} /$ Hexanes, $0.8 \mathrm{~mL} / \mathrm{min} ., 8.9 \mathrm{~min}$. (major), $14.7 \mathrm{~min}$. (minor).

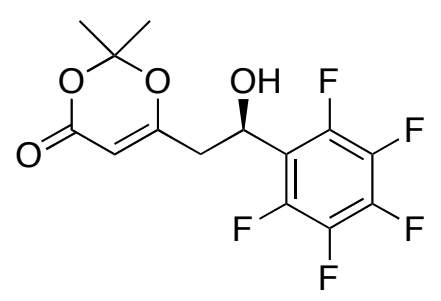

(R)-6-(2-hydroxy-2-(perfluorophenyl)ethyl)-2,2-dimethyl-4H-1,3-dioxin-4-one (3i)

Colorless oil. $\mathrm{Rf}=0.60(50 \%$ EtOAc/Hex $) ; \quad[\alpha]^{25}-2.6^{\circ}\left(c=1.00, \mathrm{CHCl}_{3}\right) ; \quad$ IR (neat, $\left.\mathrm{cm}^{-1}\right)$

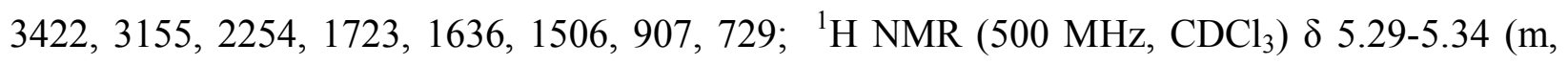
1H), $5.32(\mathrm{~s}, 1 \mathrm{H}), 3.16(\mathrm{~d}, J=6.5 \mathrm{~Hz}, 1 \mathrm{H}), 2.99(\mathrm{dd}, J=14.5,8.5 \mathrm{~Hz}, 1 \mathrm{H}), 2.74(\mathrm{dd}, J=14.5$, $5.8 \mathrm{~Hz}, 1 \mathrm{H}), 1.68(\mathrm{~s}, 3 \mathrm{H}), 1.63(\mathrm{~s}, 3 \mathrm{H}) ;{ }^{13} \mathrm{C} \mathrm{NMR}\left(125 \mathrm{MHz}, \mathrm{CDCl}_{3}\right) \delta 167.1,161.2,146.0$, 144.0, 138.8, 136.8, 107.2, 95.6, 62.7, 40.5, 25.1, 24.9; HRMS (ESI, $m / z)$ calcd for 
$\mathrm{C}_{14} \mathrm{H}_{11} \mathrm{~F}_{5} \mathrm{O}_{4} \mathrm{Na}^{+}$361.046968, found $699.104718\left(2 \mathrm{M}+\mathrm{Na}^{+}\right)$; HPLC: $10 \% i \operatorname{PrOH} /$ Hexanes, 0.8 $\mathrm{mL} / \mathrm{min} ., 10.2 \mathrm{~min}$. (major), 11.1 min. (minor).

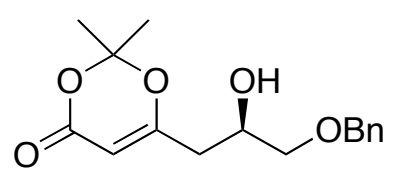

(R)-6-(3-(benzyloxy)-2-hydroxypropyl)-2,2-dimethyl-4H-1,3-dioxin-4-one (3k)

Colorless oil. $\mathrm{Rf}=0.20(50 \%$ EtOAc/Hex $) ;[\alpha]^{25}{ }_{\mathrm{D}}+3.5^{\circ}\left(c=0.50, \mathrm{CHCl}_{3}\right)$, lit.: $-15.1^{\circ}$ for the enantiomer $(92 \% \text { ee })^{3} ;{ }^{1} \mathrm{H}$ NMR $\left(400 \mathrm{MHz}, \mathrm{CDCl}_{3}\right) \delta 7.40-7.25(\mathrm{~m}, 5 \mathrm{H}), 5.33(\mathrm{~s}, 1 \mathrm{H}), 4.57(\mathrm{~s}$, 2H), 4.13-4.05 (m, 1H), 3.53 (dd, $J=9.4,3.5 \mathrm{~Hz}, 1 \mathrm{H}), 3.40$ (dd, $J=9.4,6.5 \mathrm{~Hz}, 1 \mathrm{H}), 2.42-2.39$ (m, 2H), 1.67 (s, 3H), $1.66(\mathrm{~s}, 3 \mathrm{H}) ; \quad$ HPLC: $20 \%$ iPrOH/Hexanes, $0.8 \mathrm{~mL} / \mathrm{min} ., 10.7 \mathrm{~min}$. (major), 12.7 min. (minor).

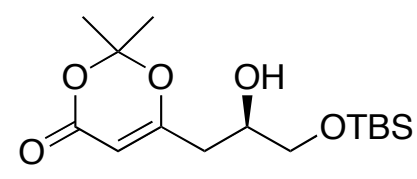

(R)-6-(3-(tert-butyldimethylsilyloxy)-2-hydroxypropyl)-2,2-dimethyl-4H-1,3-dioxin-4-one (31)

Colorless oil. $\mathrm{Rf}=0.31(30 \%$ EtOAc/Hex $) ;[\alpha]^{25}{ }_{\mathrm{D}}+4.0^{\circ}\left(c=0.30, \mathrm{CHCl}_{3}\right)$, lit.: $-15.6^{\circ}$ for the enantiomer $(>98 \% \text { ee })^{4} ;{ }^{1} \mathrm{H}$ NMR $\left(400 \mathrm{MHz}, \mathrm{CDCl}_{3}\right) \delta 5.35(\mathrm{~s}, 1 \mathrm{H}), 4.00-3.90(\mathrm{~m}, 1 \mathrm{H}), 3.67$ (dd, $J=10.0,3.7 \mathrm{~Hz}, 1 \mathrm{H}), 3.49(\mathrm{dd}, J=10.0,6.2 \mathrm{~Hz}, 1 \mathrm{H}), 2.44(\mathrm{~d}, J=5.0 \mathrm{~Hz}, 1 \mathrm{H}), 2.39-2.37$ (m, 1H), 2.37-2.35 (m, 1H), $1.70(\mathrm{~s}, 3 \mathrm{H}), 1.70(\mathrm{~s}, 3 \mathrm{H}), 0.91(\mathrm{~s}, 9 \mathrm{H}), 0.09(\mathrm{~s}, 6 \mathrm{H})$; HPLC: 5\% $i \mathrm{PrOH} / \mathrm{Hexanes}, 0.8 \mathrm{~mL} / \mathrm{min}$., $9.5 \mathrm{~min}$. (major), $11.5 \mathrm{~min}$. (minor).

\footnotetext{
${ }^{3}$ Evans, D. A.; Murry, J. A.; Kozlowski, M. C. J. Am. Chem. Soc. 1996, 118, 5814.
} 


\section{Preparation of Compounds 8b and 8d}<smiles>CCOC(=O)C(O)CC(C)=O</smiles>

\section{(R)-ethyl 2-hydroxy-4-oxopentanoate (8b)}

Water (5 drops) was added to a solution of dioxinone $3 \mathbf{b}(18.3 \mathrm{mg}, 0.07 \mathrm{mmol})$ in toluene $(2$ $\mathrm{mL}$ ). The mixture was refluxed at $120{ }^{\circ} \mathrm{C}$ for 6 hours, then concentrated in vacuo. Flash chromatography (50\% EtOAc/Hex) afforded the ketone $8 \mathbf{b}(10.4 \mathrm{mg}, 87 \%)$.

Colorless oil. $\mathrm{Rf}=0.27(50 \% \mathrm{EtOAc} / \mathrm{Hex}) ;[\alpha]^{25}+5.5^{\circ}\left(c=0.50, \mathrm{CHCl}_{3}\right) ; \quad$ IR $\left(\right.$ neat, $\left.\mathrm{cm}^{-1}\right)$ 3473, 2984, 2917, 1734, 1718, 1263; ${ }^{1} \mathrm{H}$ NMR (400 MHz, $\left.\mathrm{CDCl}_{3}\right) \delta 4.46(\mathrm{~m}, 1 \mathrm{H}), 4.25(\mathrm{q}, J=$ $7.1 \mathrm{~Hz}, 2 \mathrm{H}), 3.18(\mathrm{~d}, J=4.9 \mathrm{~Hz}, 1 \mathrm{H}), 2.98(\mathrm{dd}, J=17.4,3.9 \mathrm{~Hz}, 1 \mathrm{H}), 2.90(\mathrm{dd}, J=17.4,6.3 \mathrm{~Hz}$, 1H), $2.20(\mathrm{~s}, 3 \mathrm{H}), 1.29(\mathrm{t}, J=7.1 \mathrm{~Hz}, 3 \mathrm{H}) ;{ }^{13} \mathrm{C} \mathrm{NMR}\left(100 \mathrm{MHz}, \mathrm{CDCl}_{3}\right) \delta 207.8,175.0,67.7$, 62.7, 47.4, 31.1, 14.5 ; MS (CI, $m / z)$ calcd for $\mathrm{C}_{7} \mathrm{H}_{12} \mathrm{O}_{4}$ 160.17, found 160.9.<smiles>CCOC(=O)/C=C/C(O)CC(C)=O</smiles>

\section{$(R, E)$-ethyl 4-hydroxy-6-oxohept-2-enoate (8d)}

Water (5 drops) was added to a solution of dioxinone 3d $(11.0 \mathrm{mg}, 0.04 \mathrm{mmol})$ in toluene (2 $\mathrm{mL}$ ). The mixture was refluxed at $120^{\circ} \mathrm{C}$ for 6 hours, then concentrated in vacuo. Flash chromatography (50\% EtOAc/Hex) afforded the ketone 8d (6.7 mg, 88\%).

Yellow oil. $\mathrm{Rf}=0.36(50 \%$ EtOAc/Hex $) ;[\alpha]_{\mathrm{D}}^{25}+20.7^{\circ}\left(c=0.67, \mathrm{CHCl}_{3}\right) ; \quad \mathrm{IR}\left(\right.$ neat, $\left.\mathrm{cm}^{-1}\right) 3476$,

\footnotetext{
${ }^{4}$ Sugita, Y.; Sakaki, J.-i.; Sato, M.; Kaneko, C. J. Chem. Soc. Perkin Trans. 1 1992, 2855.
} 
2982, 2925, 1717, 1660, 1306; ${ }^{1} \mathrm{H}$ NMR (400 MHz, $\left.\mathrm{CDCl}_{3}\right) \delta 6.87$ (ddd, $J=15.7,4.2,0.4 \mathrm{~Hz}$, $1 \mathrm{H}), 6.11(\mathrm{dd}, J=15.6,1.9 \mathrm{~Hz}, 1 \mathrm{H}), 4.80-4.70(\mathrm{~m}, 1 \mathrm{H}), 4.20(\mathrm{q}, J=7.1 \mathrm{~Hz}, 2 \mathrm{H}), 3.24(\mathrm{dd}, J=$ 4.1, $0.4 \mathrm{~Hz}, 1 \mathrm{H}), 2.76(\mathrm{dd}, J=17.9,3.3 \mathrm{~Hz}, 1 \mathrm{H}), 2.65(\mathrm{dd}, J=17.9,8.8 \mathrm{~Hz}, 1 \mathrm{H}), 2.20(\mathrm{~s}, 3 \mathrm{H})$, $1.28(\mathrm{t}, J=7.1 \mathrm{~Hz}, 3 \mathrm{H}) ;{ }^{13} \mathrm{C} \mathrm{NMR}\left(100 \mathrm{MHz}, \mathrm{CDCl}_{3}\right) \delta 209.9,167.7,148.7,122.0,67.4,61.2$, 49.3, 31.2, 14.7; HRMS (ESI, $m / z$ ) calcd for $\mathrm{C}_{9} \mathrm{H}_{14} \mathrm{O}_{4} \mathrm{Na}^{+}$209.078428, found 395.167638 $\left(2 \mathrm{M}+\mathrm{Na}^{+}\right)$.

Determination of the absolute configuration of $8 \mathrm{~b}$

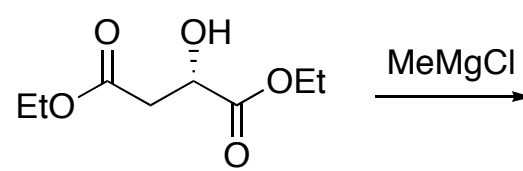

(S)-9

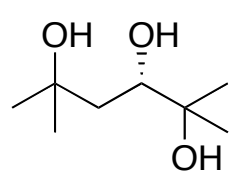

(S)-10

\section{(S)-2,5-dimethylhexane-2,3,5-triol ((S)-10)}

(S)-9 was prepared in one step from L-malic acid. ${ }^{5}$ A solution of $(\boldsymbol{S})-9$ (35 $\left.\mathrm{mg}, 0.18 \mathrm{mmol}\right)$ in THF $(1 \mathrm{~mL})$ was added dropwise to a solution of $\mathrm{MeMgCl}(3.0 \mathrm{M}$ in THF, $0.61 \mathrm{~mL}, 1.8 \mathrm{mmol})$ in THF $(1 \mathrm{~mL})$ at $-78^{\circ} \mathrm{C}$. The cooling bath was removed and the resulting solution was stirred at room temperature for 1 hour, after which the reaction was carefully quenched with water at $0{ }^{\circ} \mathrm{C}$. The reaction mixture was extracted with $\mathrm{Et}_{2} \mathrm{O}(3 \times 10 \mathrm{~mL})$, dried over $\mathrm{MgSO}_{4}$ and concentrated in vacuo. Flash chromatography (70\% EtOAc/Hex) afforded the triol (S)-10 (12 mg, 42\%).

White solid. mp 52-54 ${ }^{\circ} \mathrm{C}$; Rf=0.22 (70\% EtOAc/Hex); $[\alpha]^{28}{ }_{\mathrm{D}}-7.7^{\circ}\left(c=0.99, \mathrm{CHCl}_{3}\right)$; IR (film cast, $\left.\mathrm{cm}^{-1}\right) 3380,2973,1381,1150 ;{ }^{1} \mathrm{H}$ NMR (400 MHz, $\left.\mathrm{CDCl}_{3}\right) \delta 3.75(\mathrm{dd}, J=11.0,2.2 \mathrm{~Hz}$,

\footnotetext{
${ }^{5}$ Meyers, A.I.; Lawson, J.P.; Walker, D.G.; Linderman, R.J.; J. Org. Chem. 1986, 51, 5111.
} 
1H), 3.09 (br s, 3H), 1.63 (dd, $J=14.4,11.0 \mathrm{~Hz}, 1 \mathrm{H}), 1.50$ (dd, $J=14.4,2.2 \mathrm{~Hz}, 1 \mathrm{H}), 1.31$ (s, 3H), $1.28(\mathrm{~s}, 3 \mathrm{H}), 1.18(\mathrm{~s}, 3 \mathrm{H}), 1.14(\mathrm{~s}, 3 \mathrm{H}) ;{ }^{13} \mathrm{C} \mathrm{NMR}\left(100 \mathrm{MHz}, \mathrm{CDCl}_{3}\right) \delta$ 76.7, 73.5, 72.4, 42.6, 32.8, 28.1, 26.9, 24.1; HRMS (ESI, $m / z$ ) calcd for $\mathrm{C}_{8} \mathrm{H}_{18} \mathrm{O}_{3} \mathrm{Na}^{+}$185.11481, found 185.11423; GC: chiral Cyclosilb column $\left(100{ }^{\circ} \mathrm{C}\right.$ to $200{ }^{\circ} \mathrm{C}$ at $15{ }^{\circ} \mathrm{C} / \mathrm{min}$.), $5.93 \mathrm{~min}$. (major), $6.02 \mathrm{~min}$. (minor).

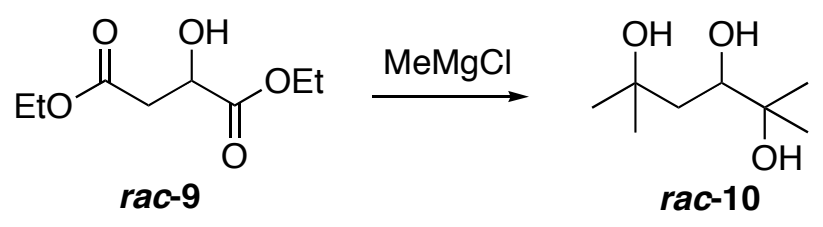

rac-2,5-dimethylhexane-2,3,5-triol (rac-10)

This compound was prepared in the same manner as $(\boldsymbol{S})$-10 above. (40\% yield).

GC: chiral cyclosilb column $\left(100{ }^{\circ} \mathrm{C}\right.$ to $200{ }^{\circ} \mathrm{C}$ at $15{ }^{\circ} \mathrm{C} / \mathrm{min}$.), $5.94 \mathrm{~min} .(S), 6.01 \mathrm{~min}$. (R).

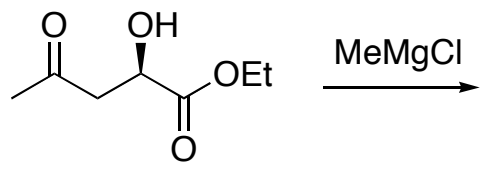

8b

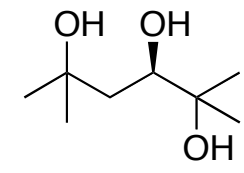

(R)-10

\section{(R)-2,5-dimethylhexane-2,3,5-triol $((R)-10)$}

This compound was prepared in the same manner as $(\boldsymbol{S})$-10 above. (40\% yield).

$[\alpha]^{28}+6.4^{\circ}\left(c=0.91, \mathrm{CHCl}_{3}\right) ; \mathrm{GC}$ : chiral cyclosilb column $\left(100{ }^{\circ} \mathrm{C}\right.$ to $200^{\circ} \mathrm{C}$ at $15{ }^{\circ} \mathrm{C} / \mathrm{min}$. $)$, 5.95 min. (minor), 6.02 min. (major) 


\section{${ }^{1} \mathrm{H}$ and ${ }^{13} \mathrm{C}$ Spectra}
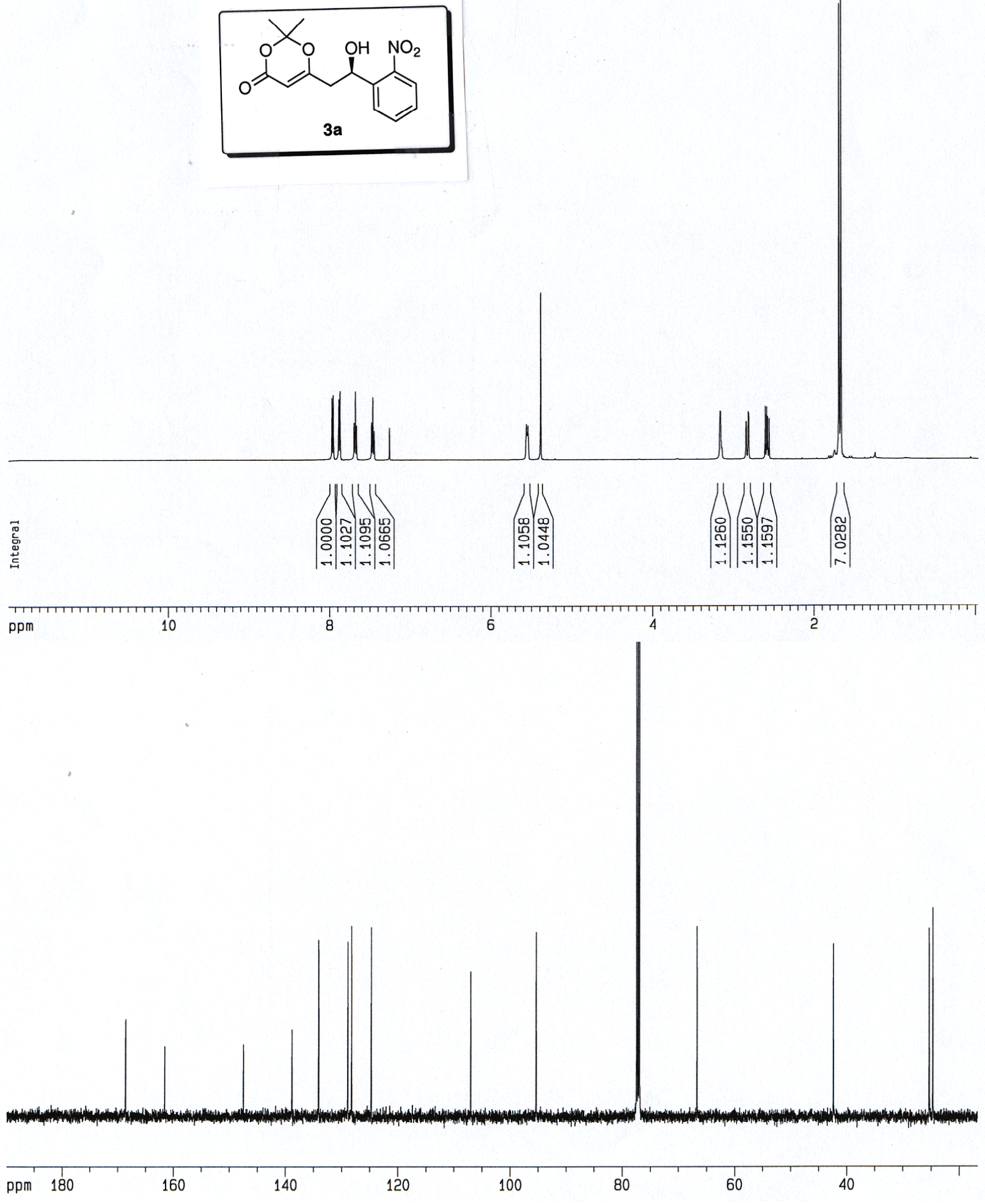
S-14
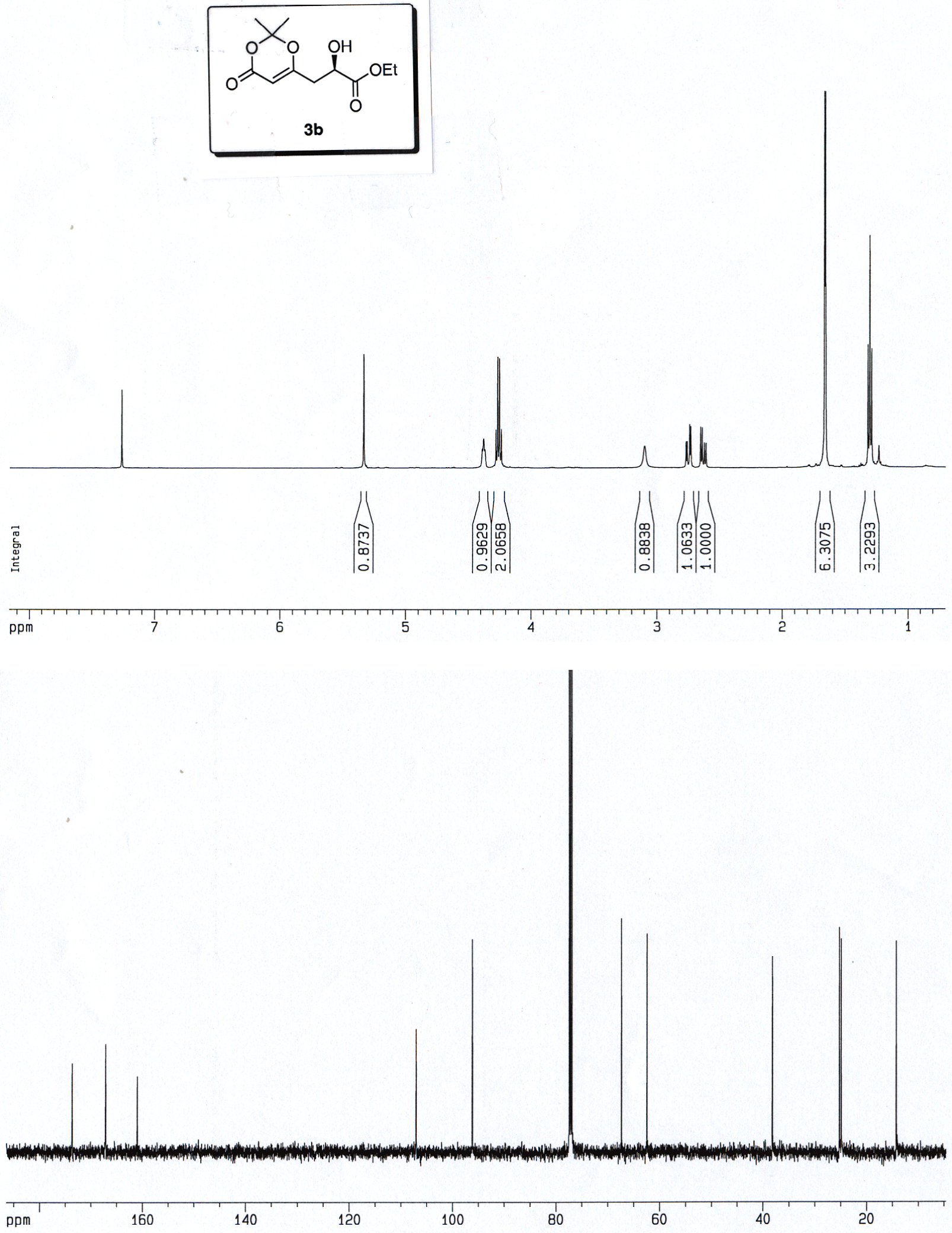


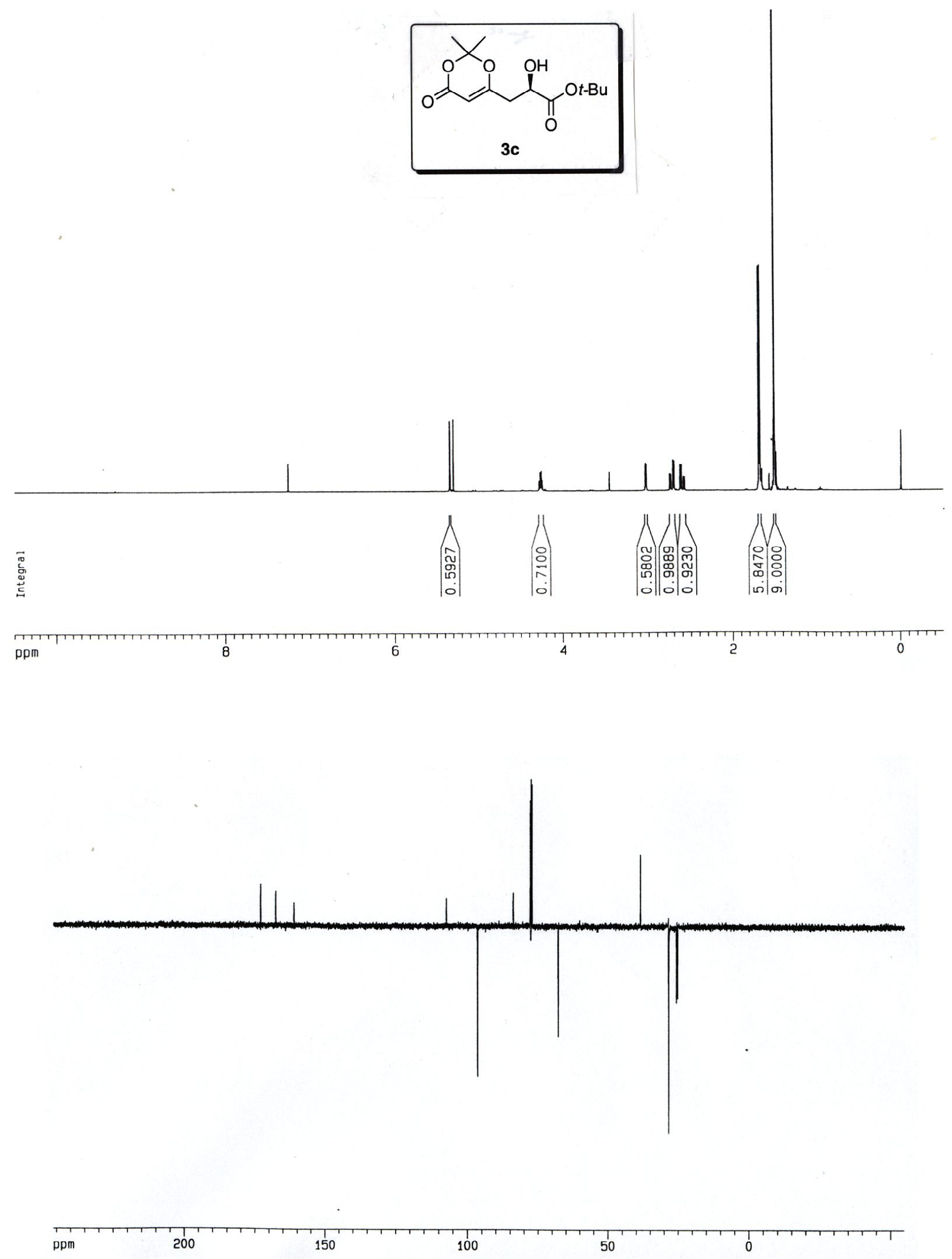


S-16
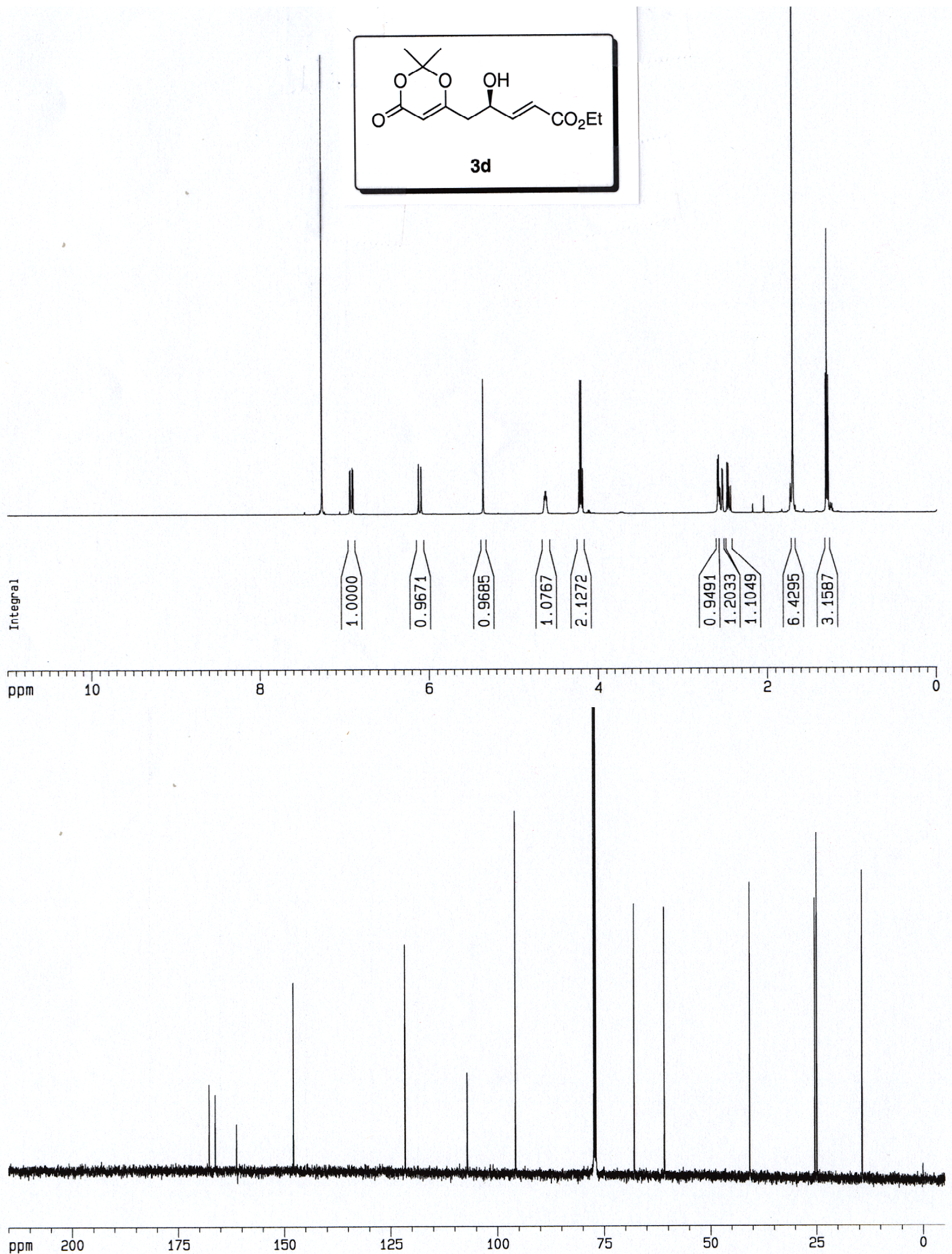

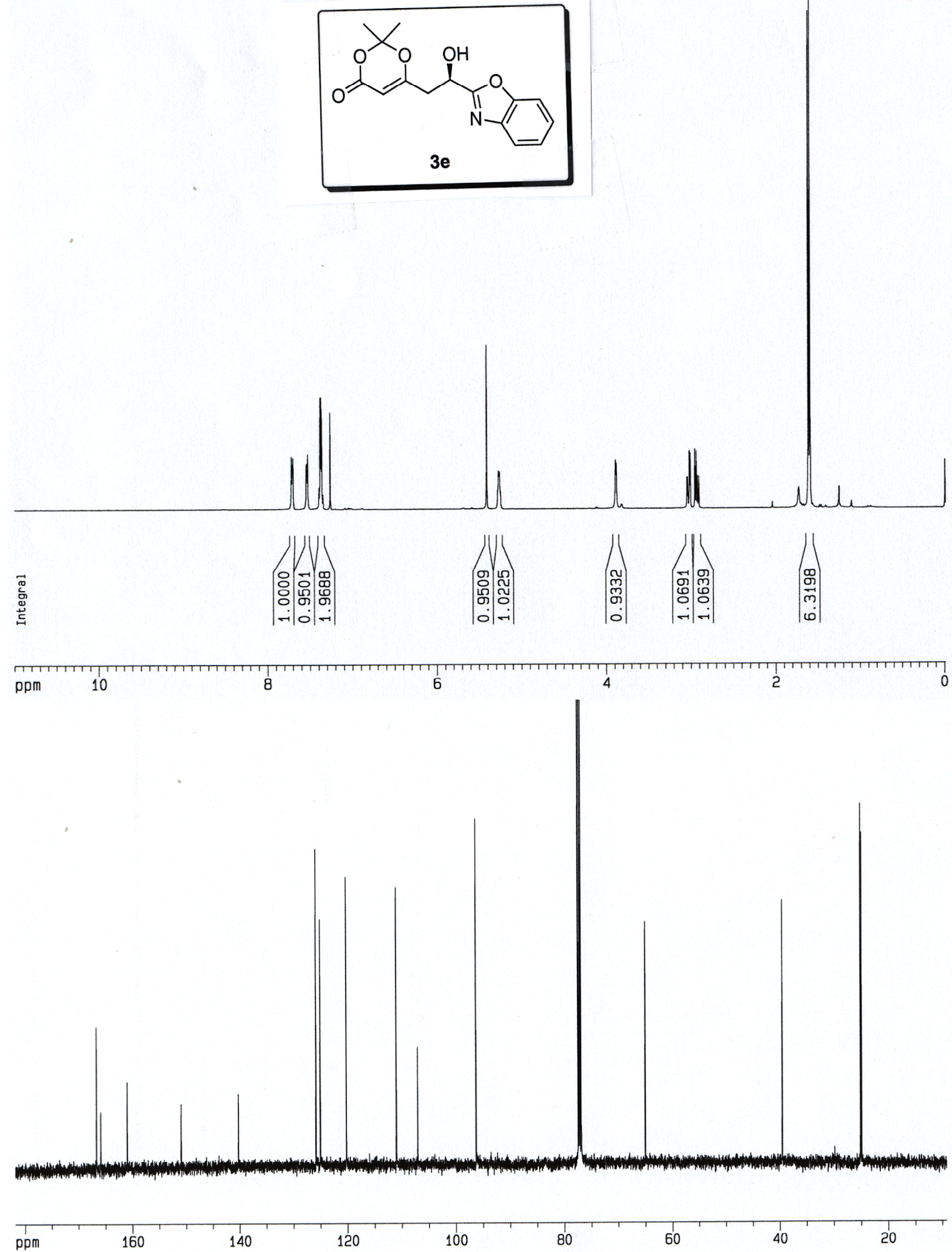

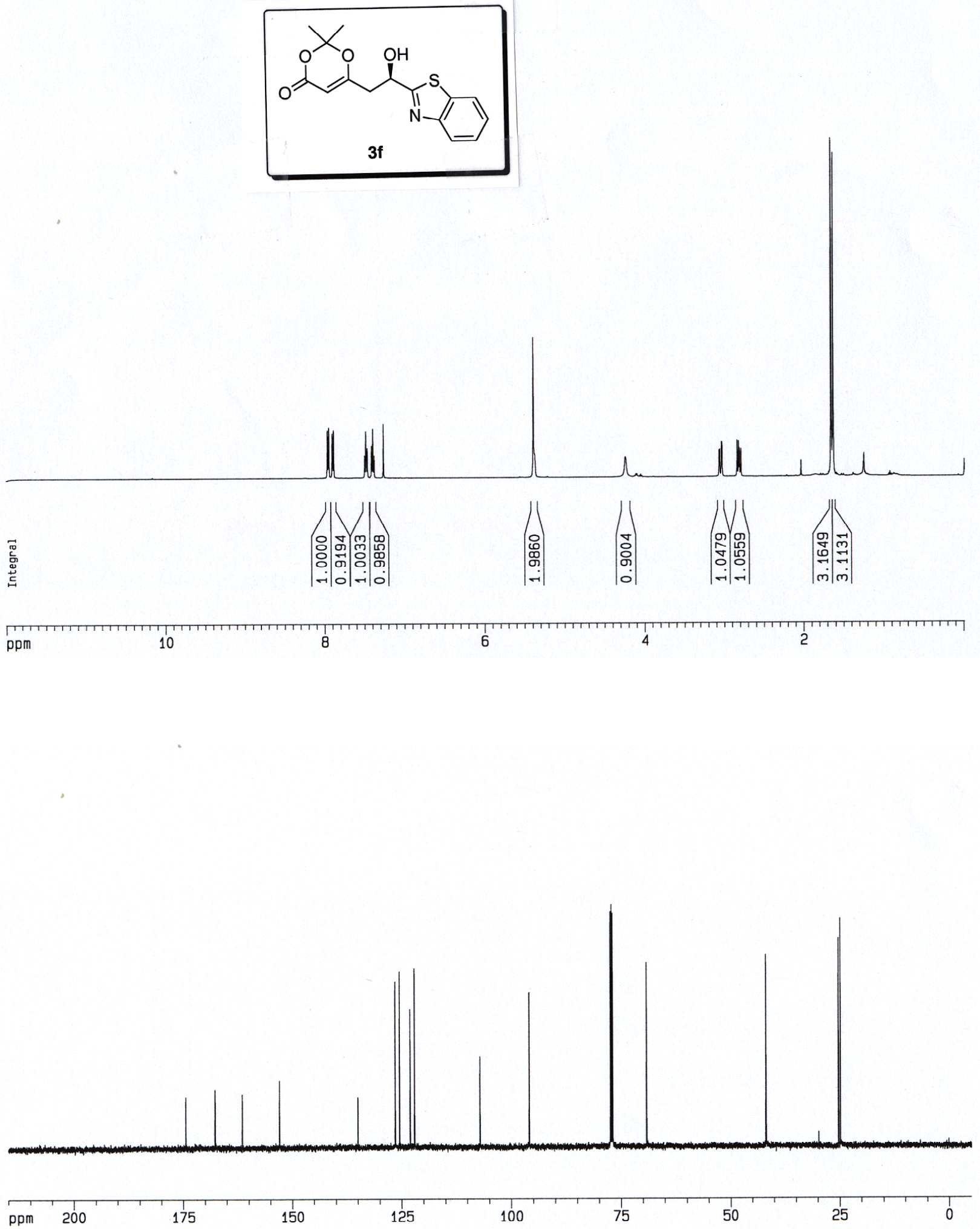

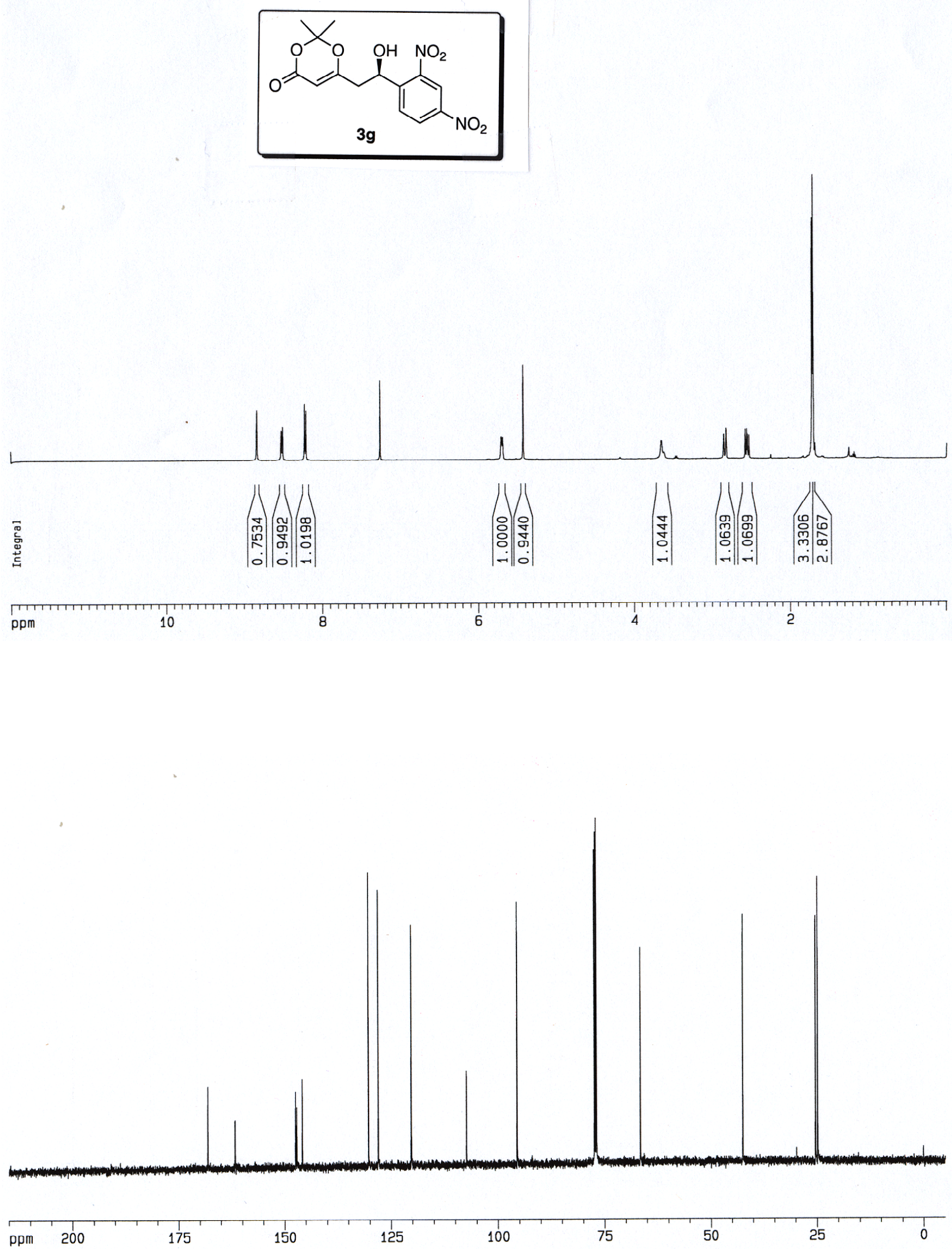

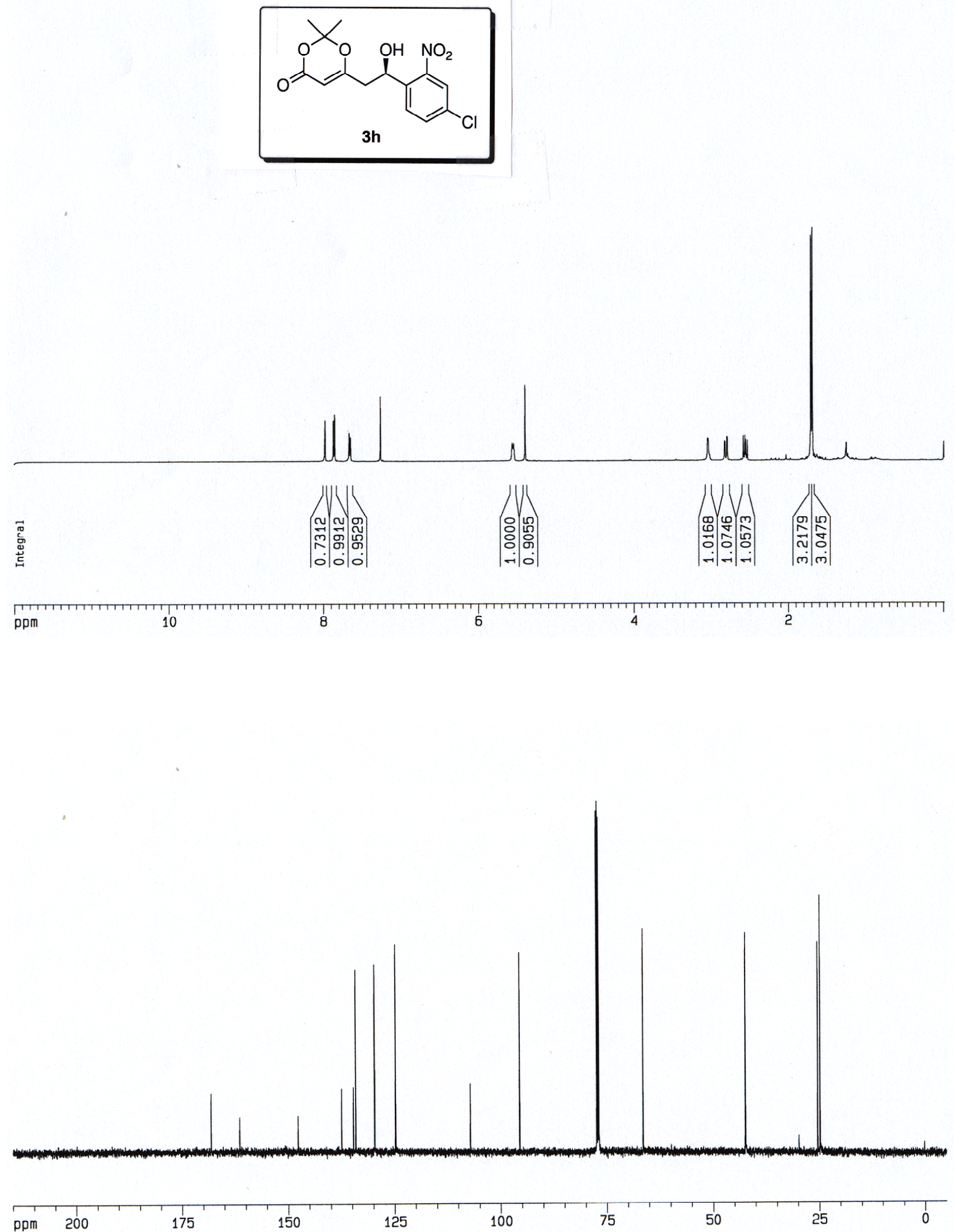

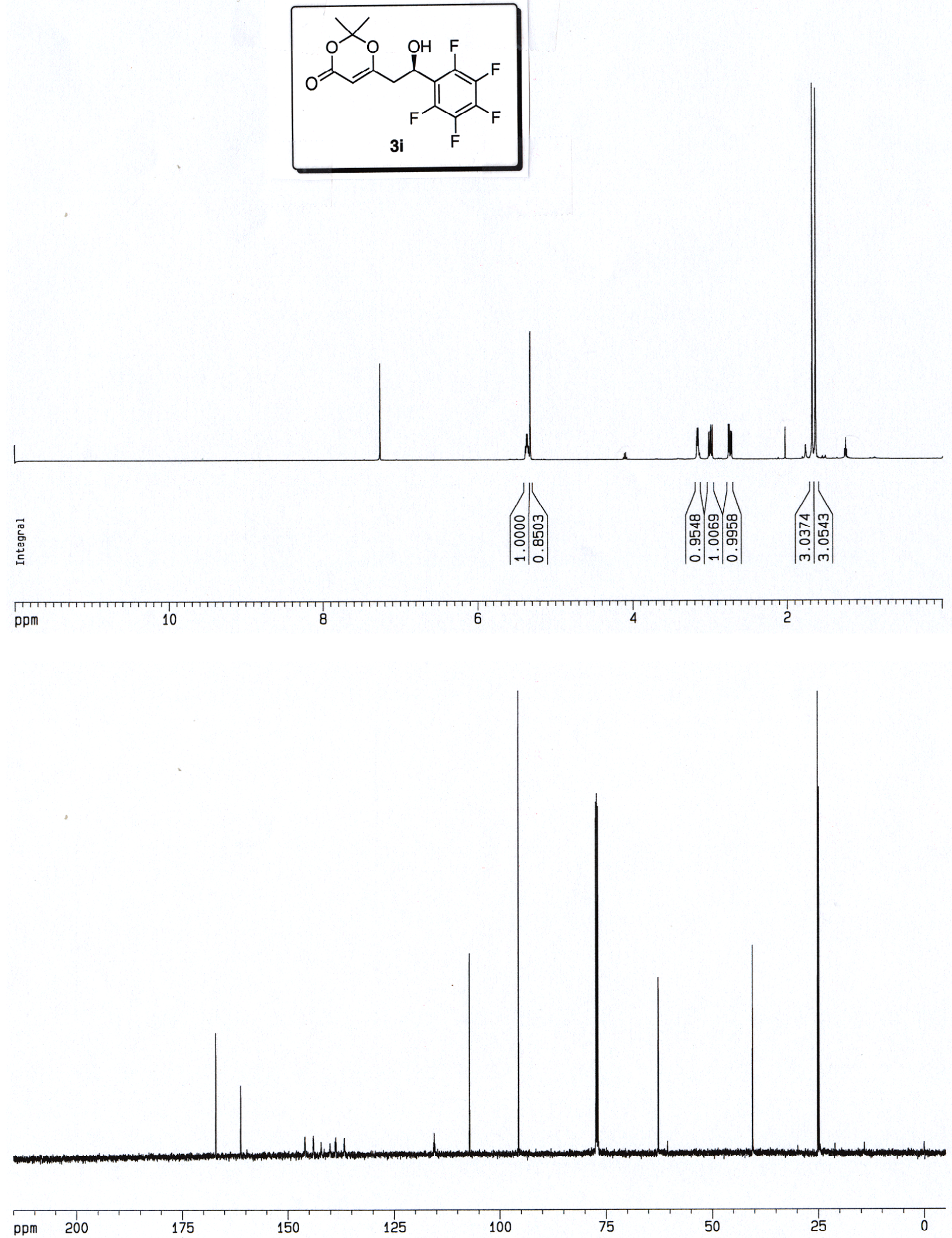

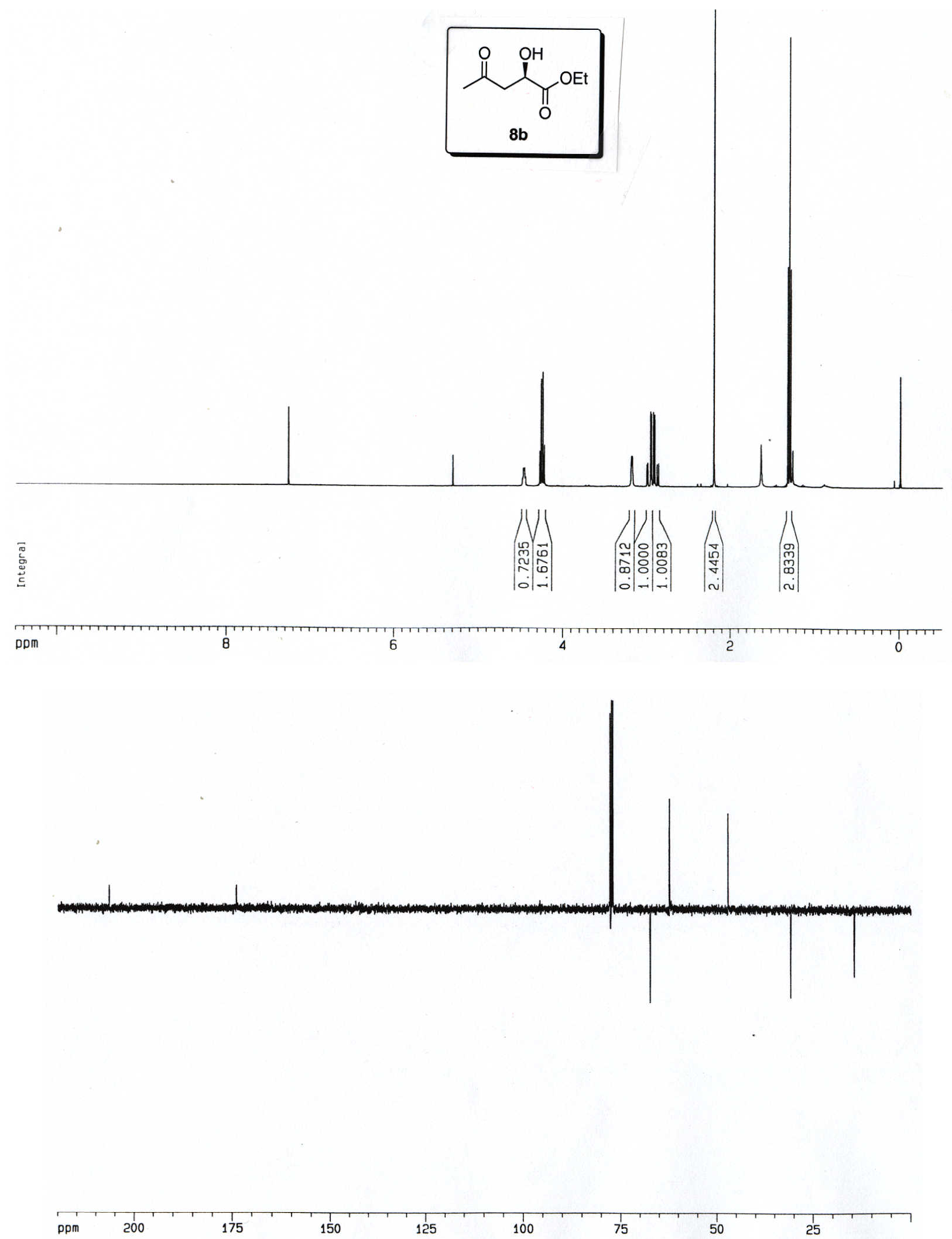


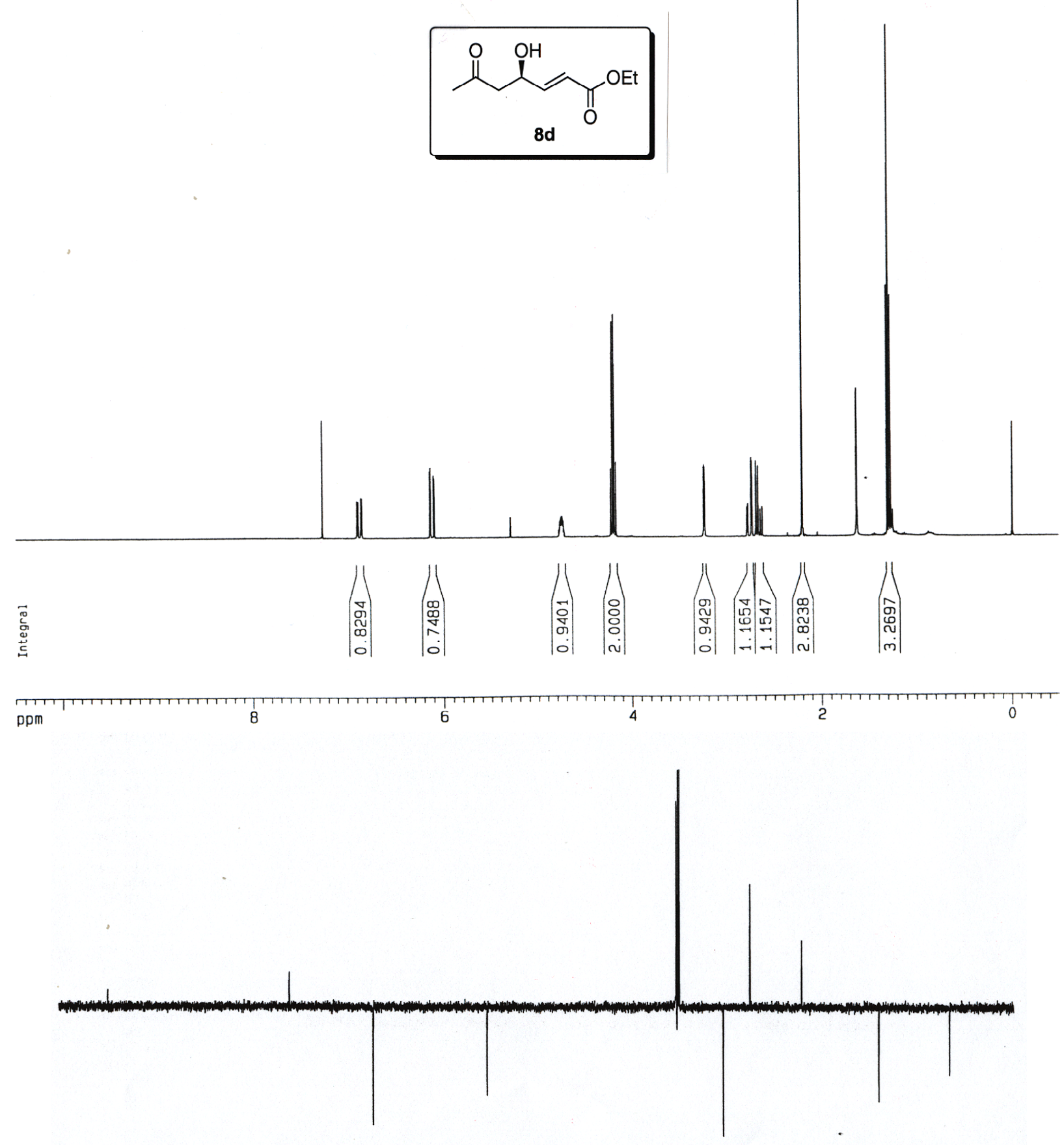

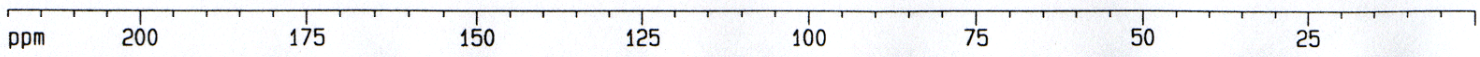



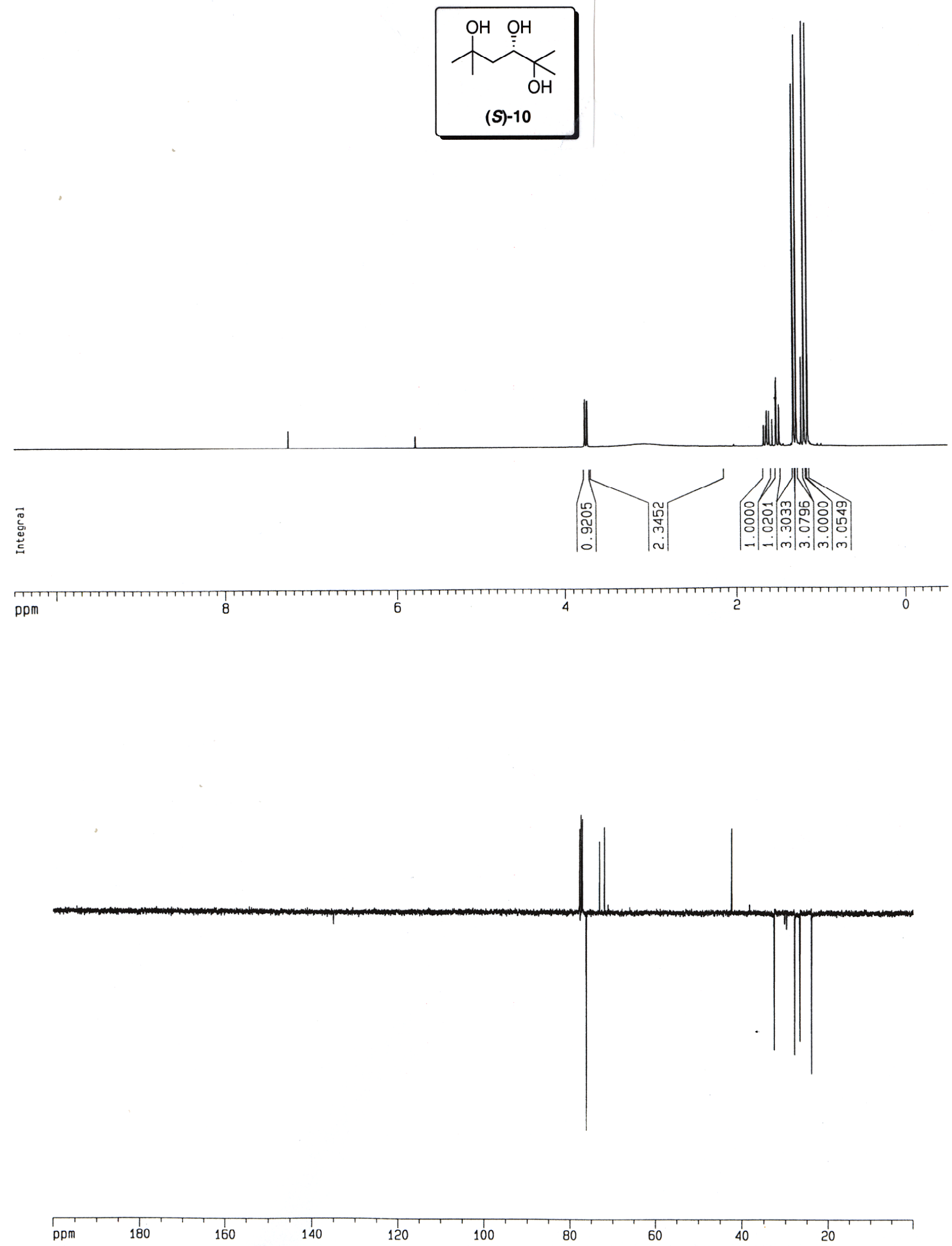


\section{HPLC Chromatograms}

$3 a$

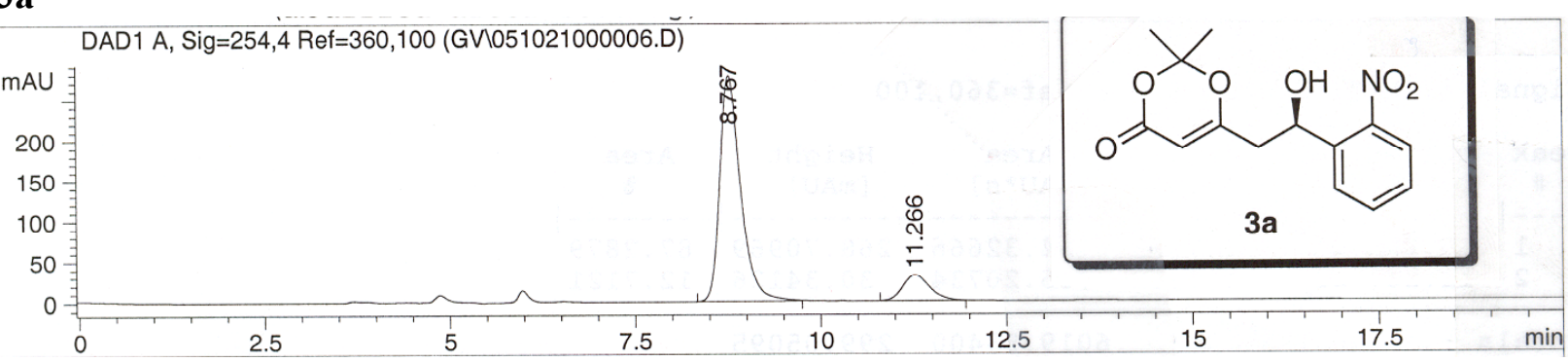

\section{Racemate:}

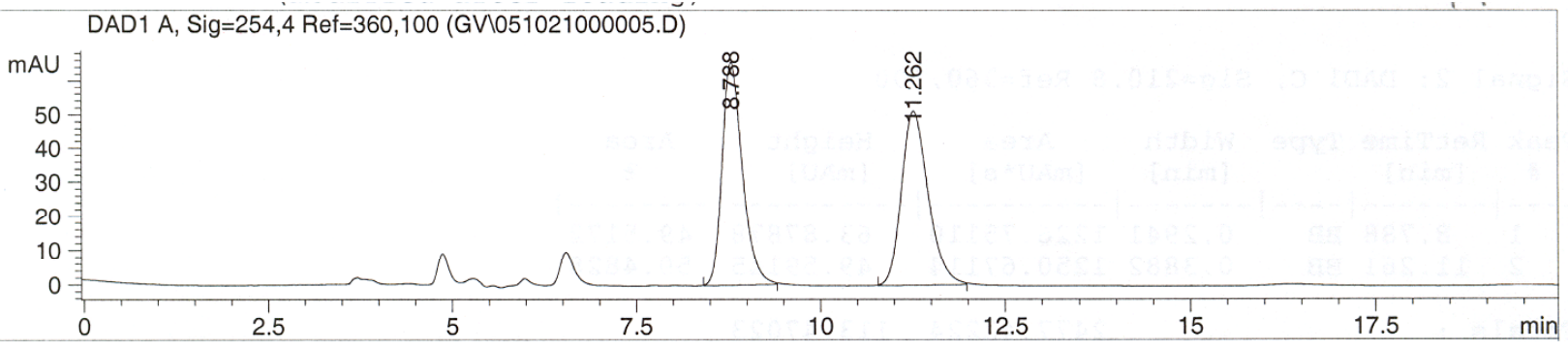

\section{3b}

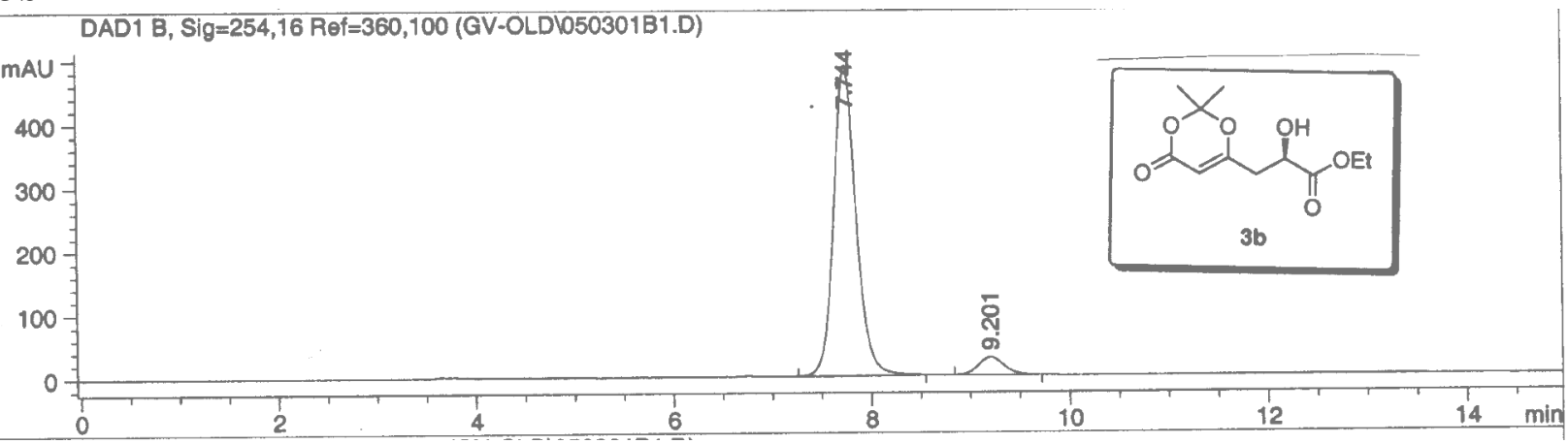

\section{Racemate:}

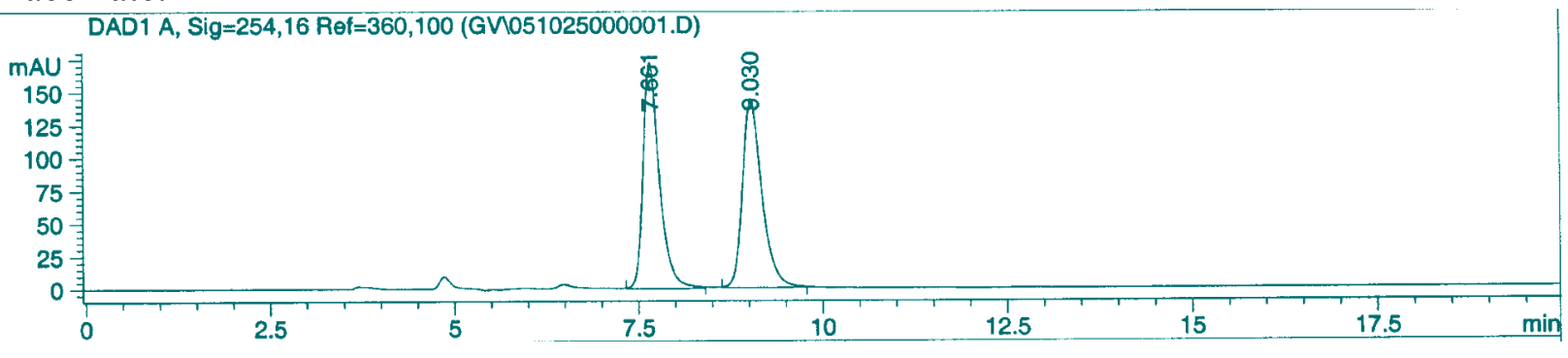


$3 c$

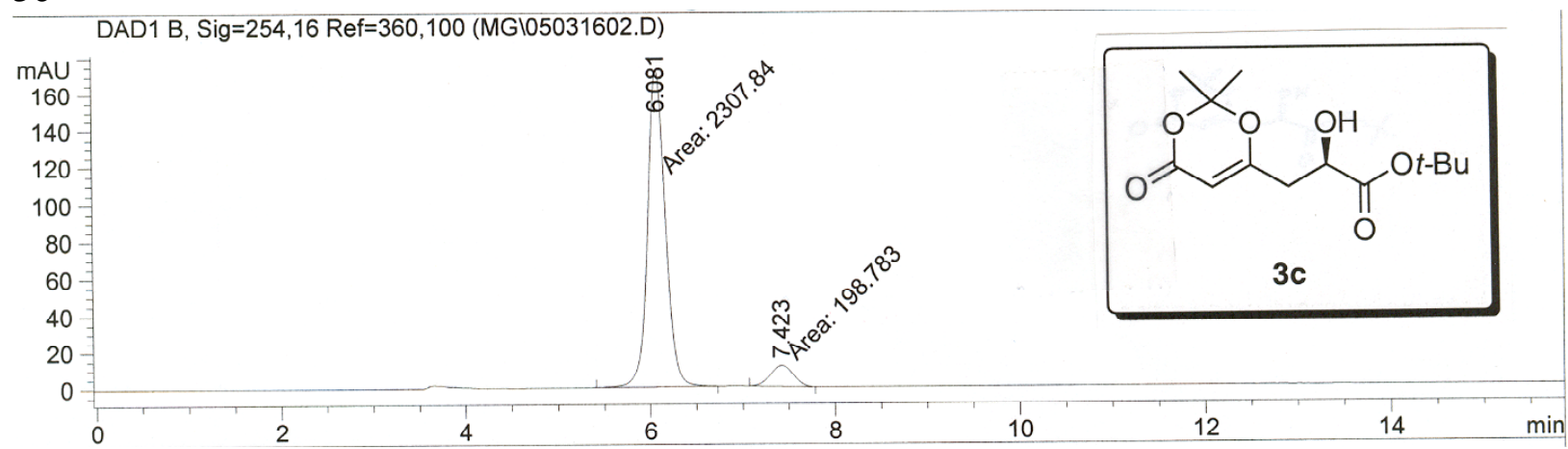

Racemate:

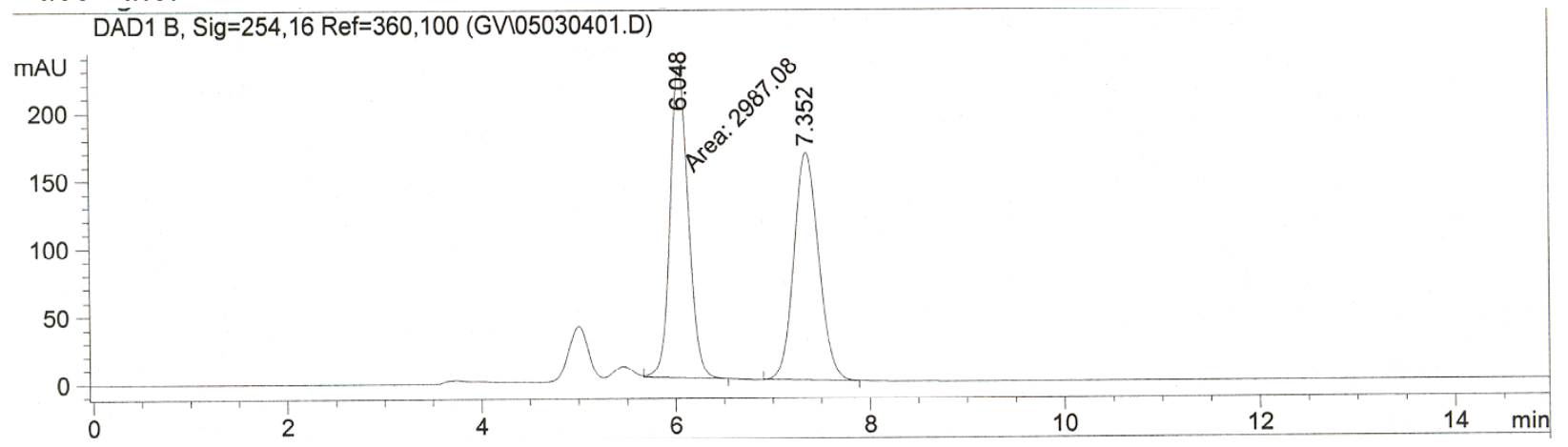

3d

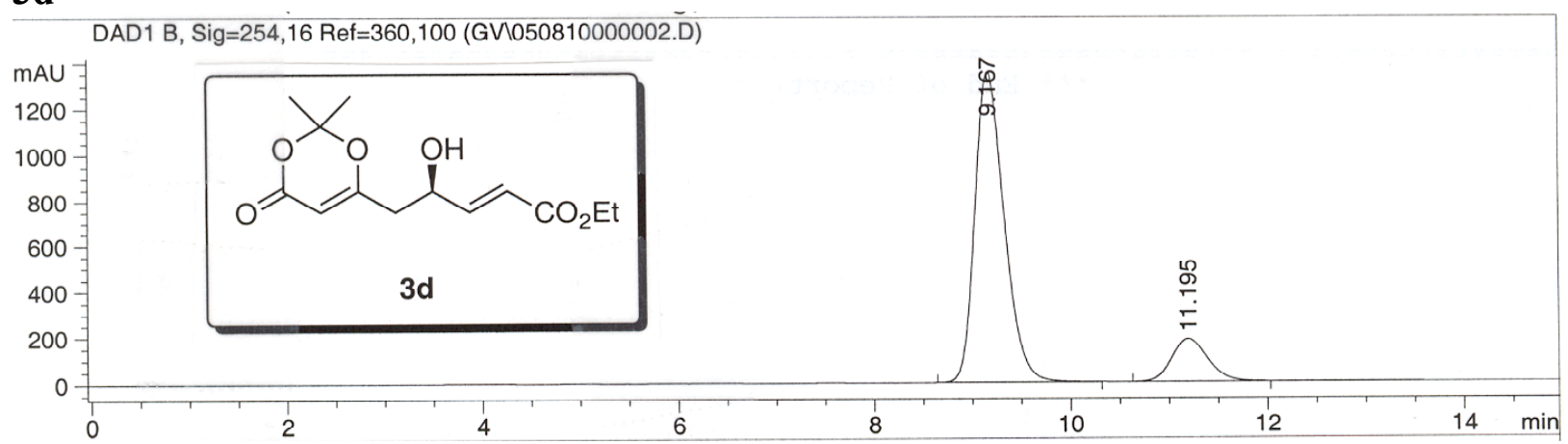

Racemate:

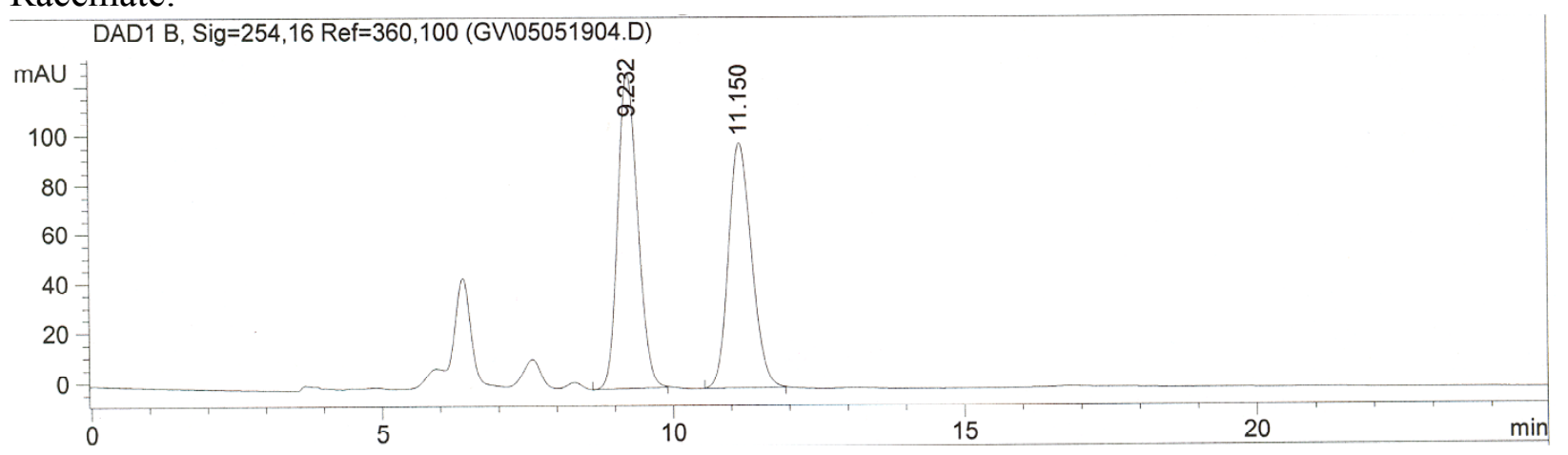




\section{$3 e$}

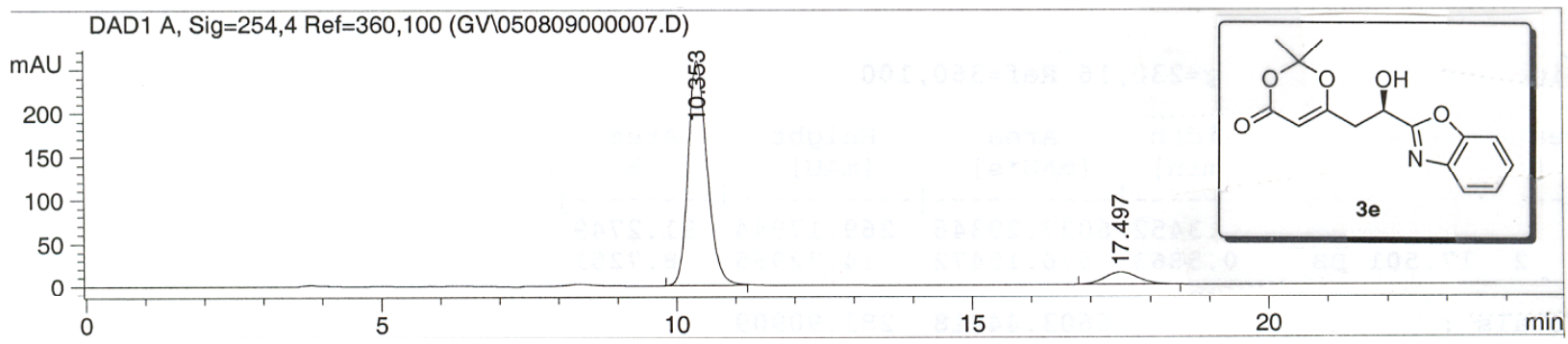

Racemate:

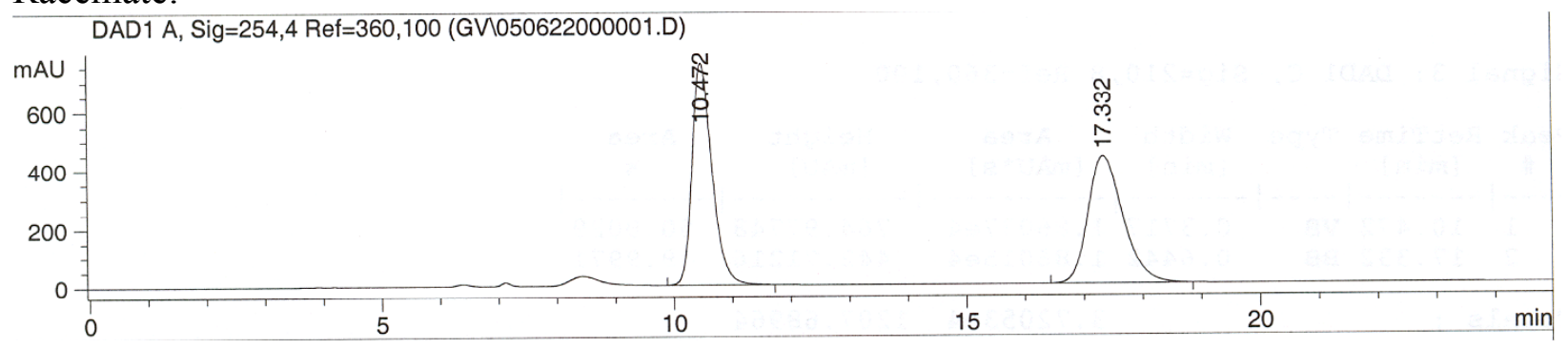

\section{3f}

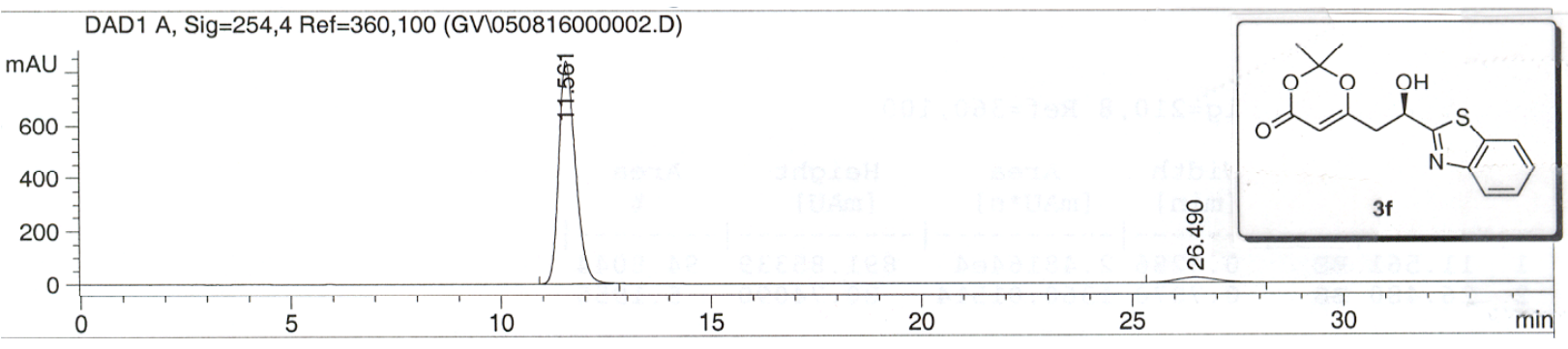

\section{Racemate:}

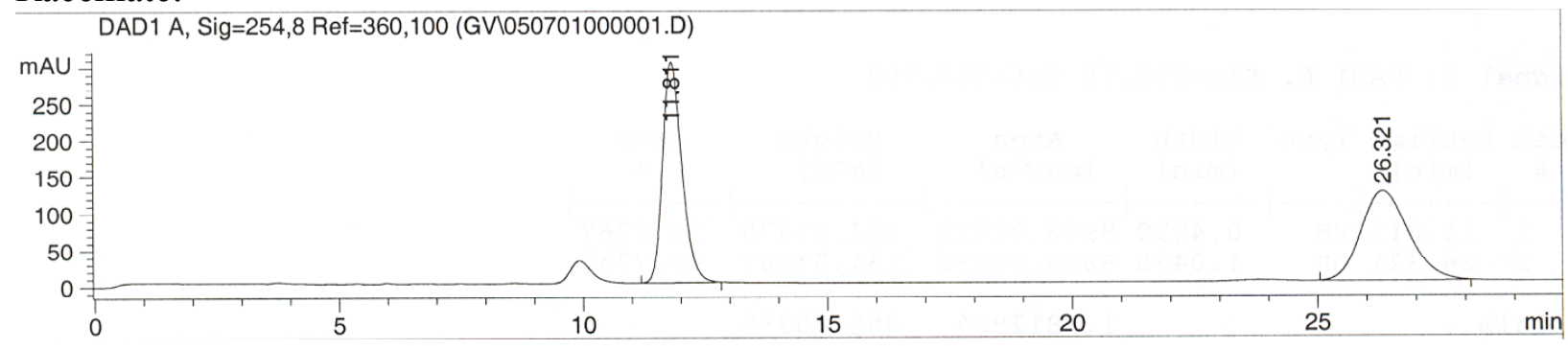


$3 g$

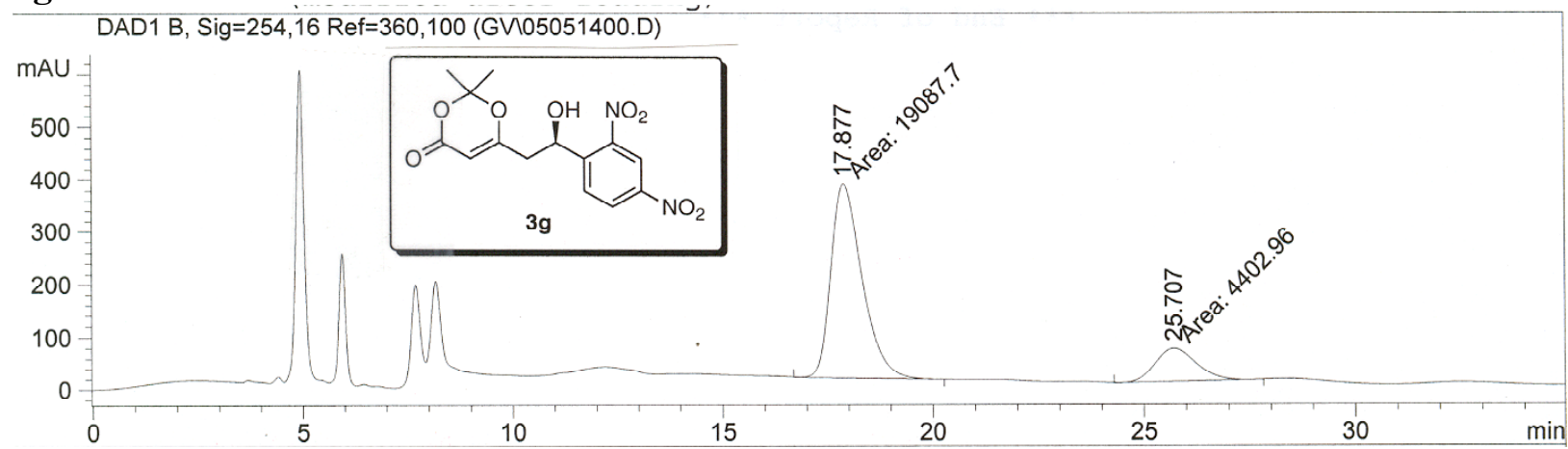

Racemate:

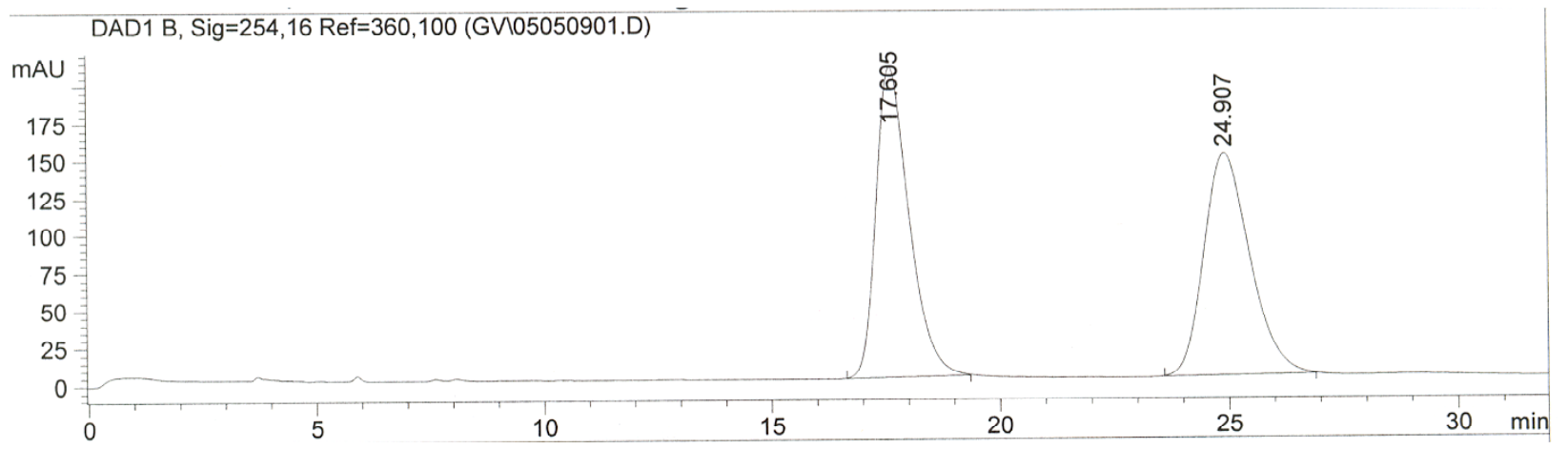

3h

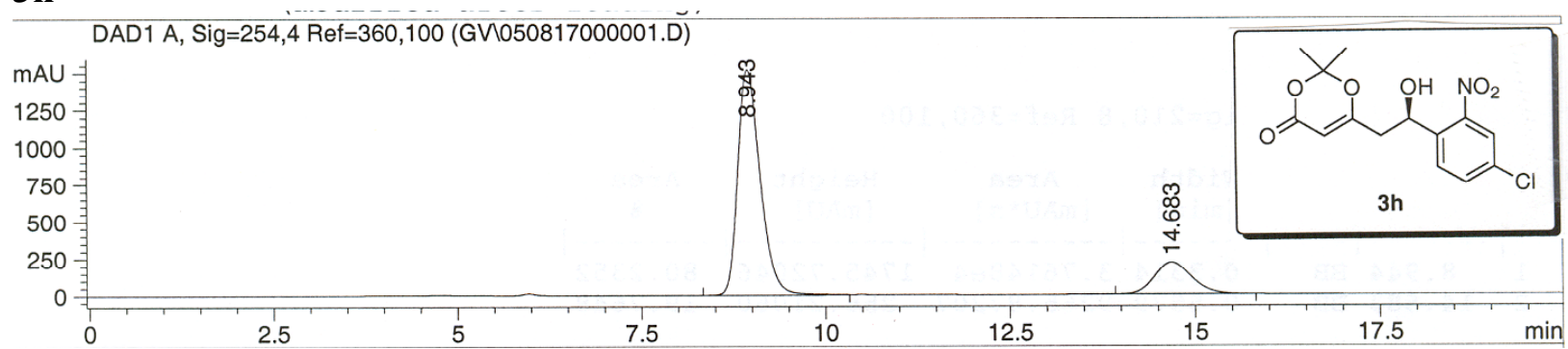

Racemate:

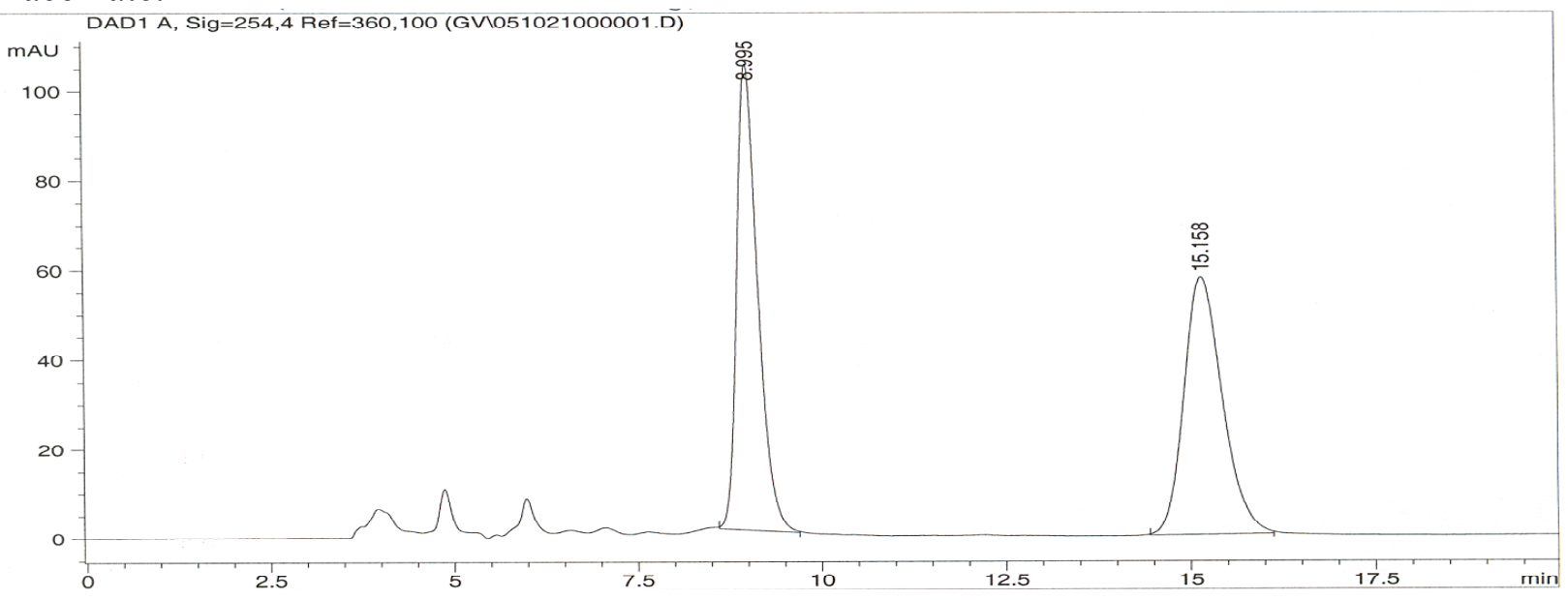




\section{$3 i$}

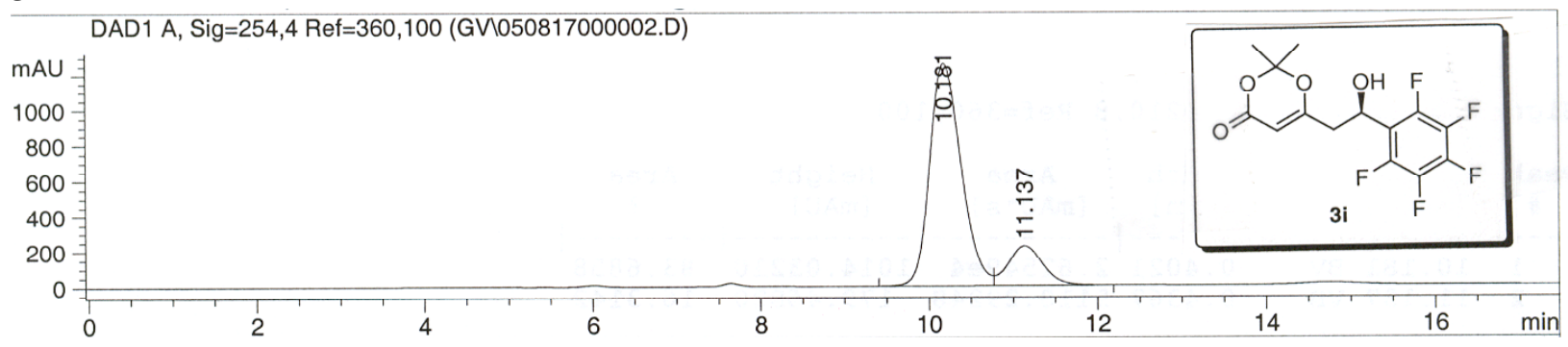

\section{Racemate:}

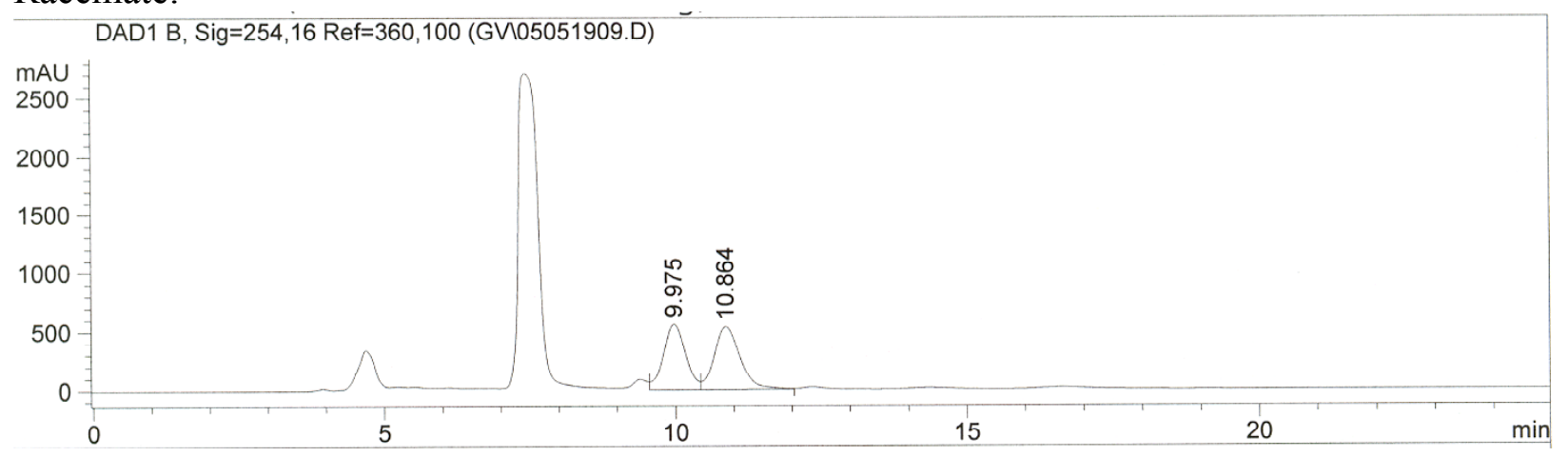

3k

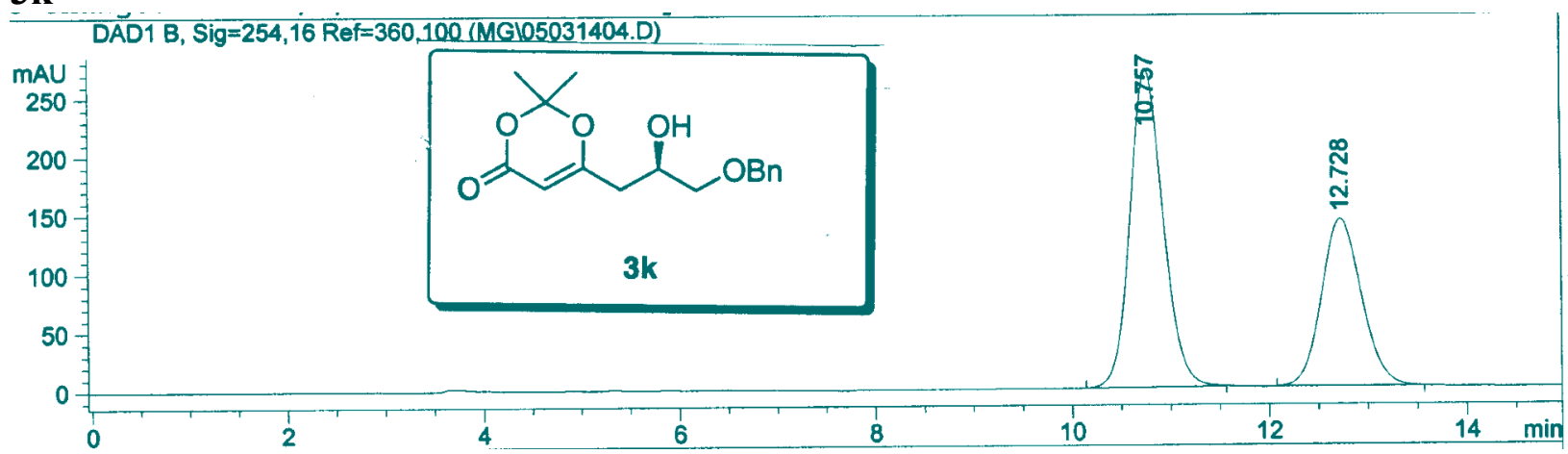

\section{Racemate:}

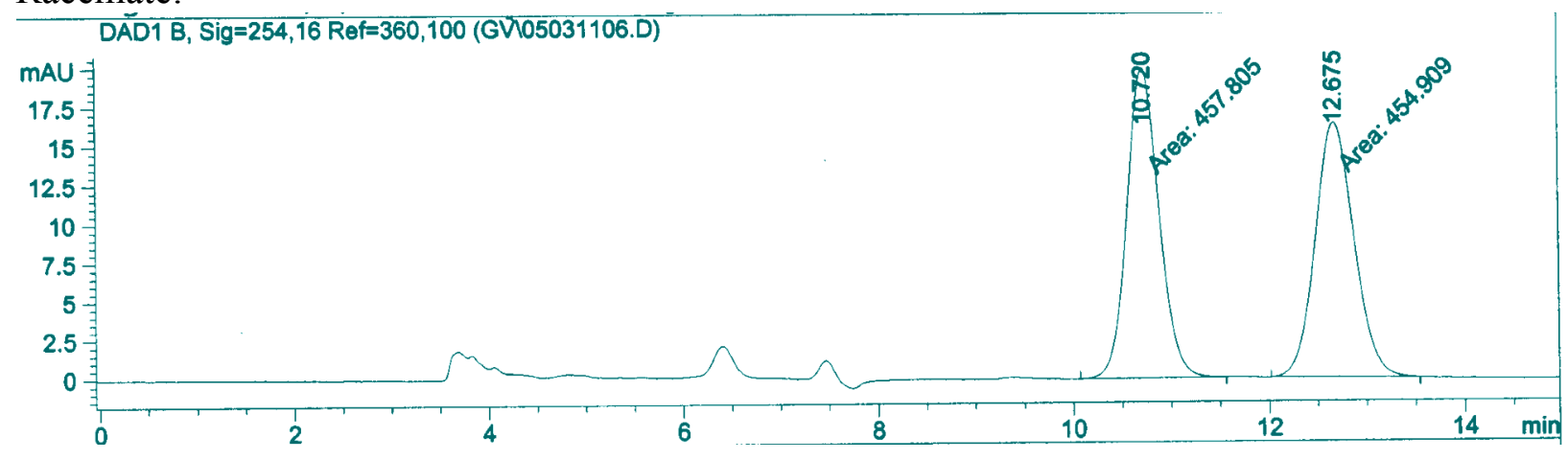




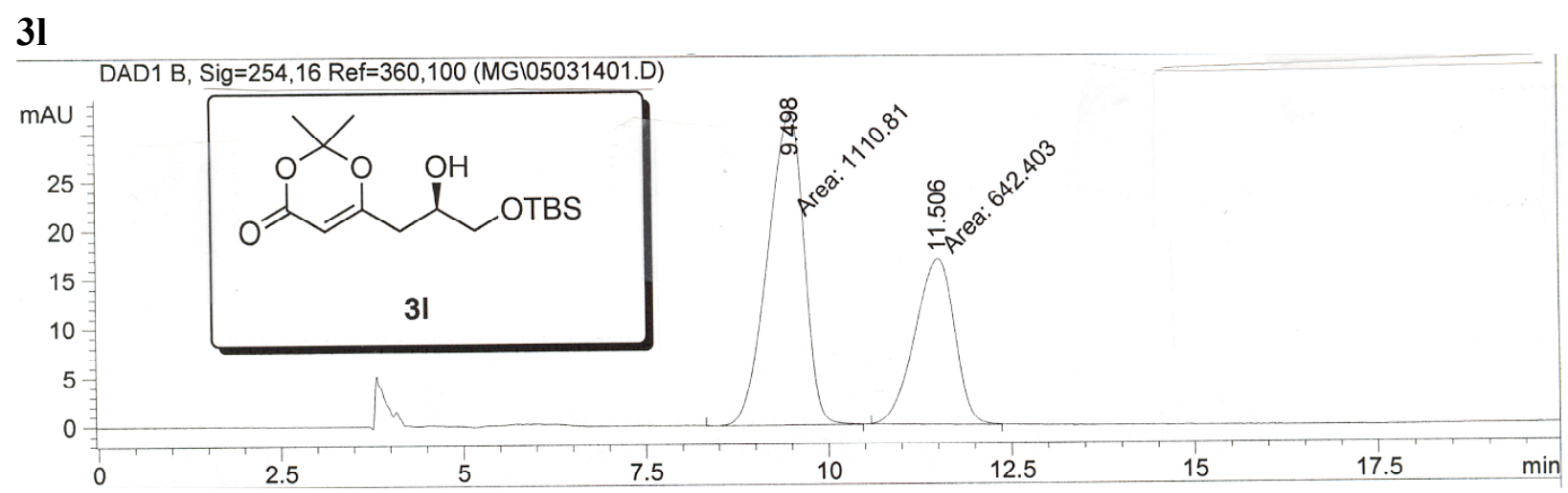

Racemate:

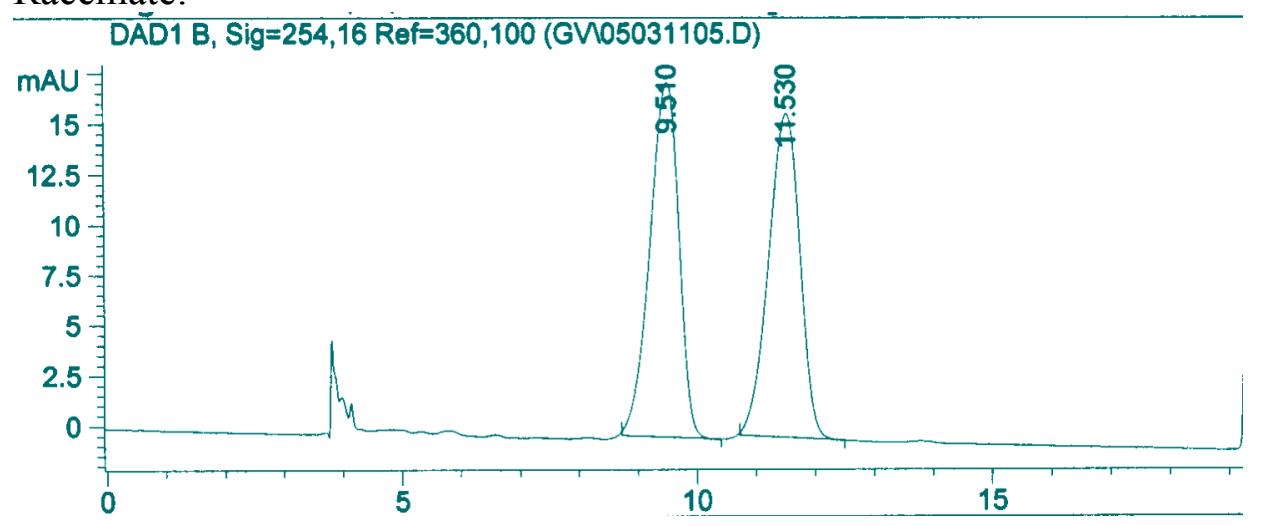

\section{GC Chromatograms}

\section{(R)-10 from 3b}

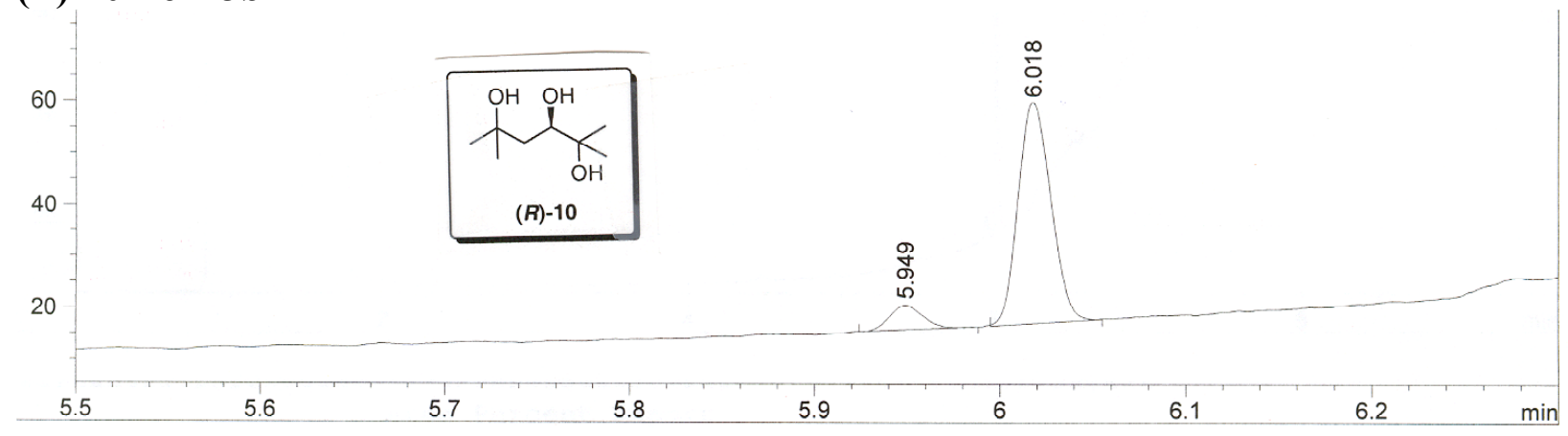


(S)-10 from L-malic acid

FID1 A, (MICHELI050916000002.D)

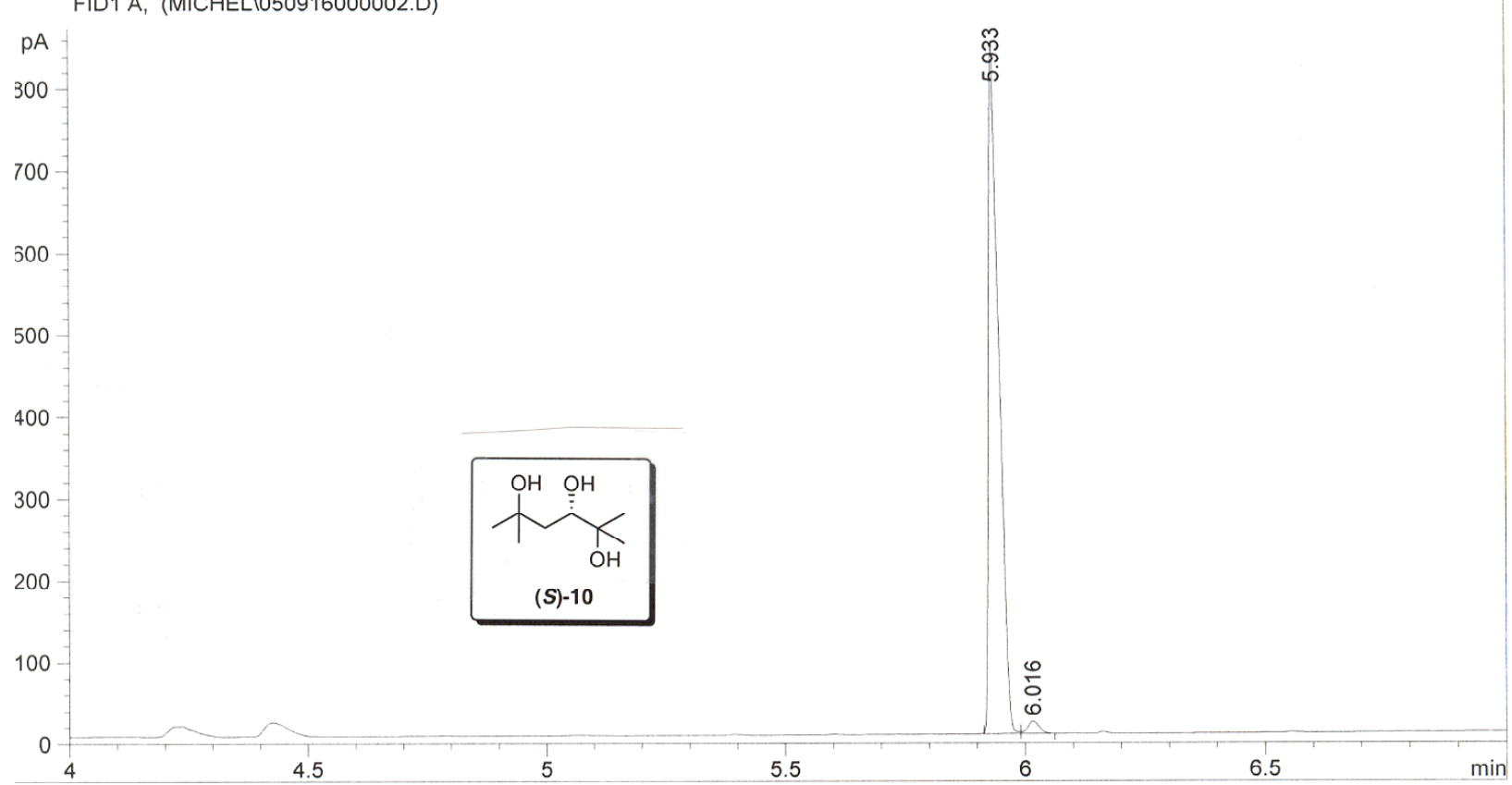

Racemate from DL-malic acid:

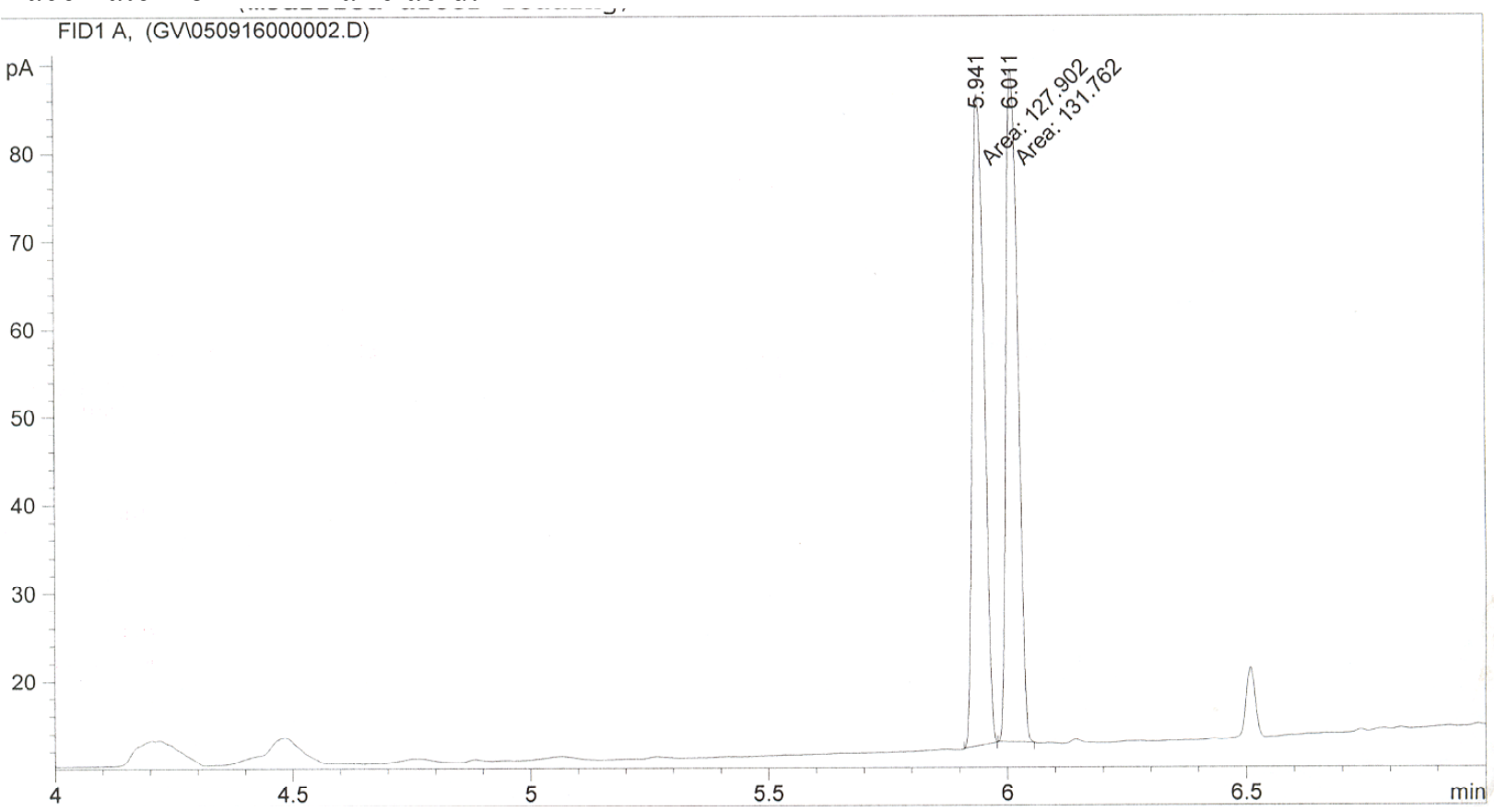

